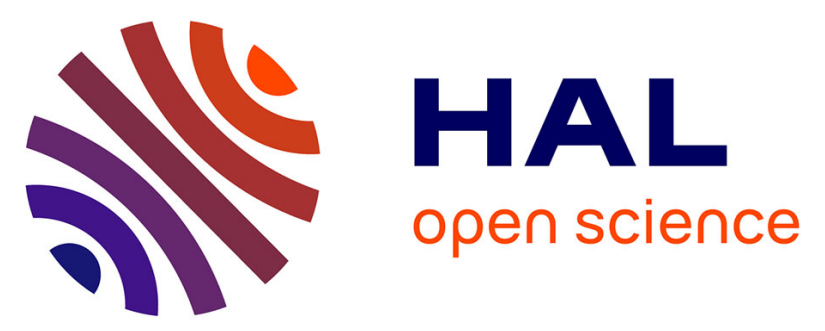

\title{
Modeling the time-dependent electron dynamics in dielectric materials induced by two-color femtosecond laser pulses: Applications to material modifications
}

P. González de Alaiza Martínez, E. Smetanina, I. Thiele, B. Chimier, G. Duchateau

\section{- To cite this version:}

P. González de Alaiza Martínez, E. Smetanina, I. Thiele, B. Chimier, G. Duchateau. Modeling the time-dependent electron dynamics in dielectric materials induced by two-color femtosecond laser pulses: Applications to material modifications. Physical Review A, 2021, 103 (3), 10.1103/PhysRevA.103.033107 . hal-03394805

\section{HAL Id: hal-03394805 \\ https://hal.science/hal-03394805}

Submitted on 22 Oct 2021

HAL is a multi-disciplinary open access archive for the deposit and dissemination of scientific research documents, whether they are published or not. The documents may come from teaching and research institutions in France or abroad, or from public or private research centers.
L'archive ouverte pluridisciplinaire HAL, est destinée au dépôt et à la diffusion de documents scientifiques de niveau recherche, publiés ou non, émanant des établissements d'enseignement et de recherche français ou étrangers, des laboratoires publics ou privés. 


\title{
Modeling the time-dependent electron dynamics in dielectric materials induced by two-color femtosecond laser pulses: Applications to material modifications
}

\author{
P. González de Alaiza Martínez, ${ }^{1}$ E. Smetanina, ${ }^{2}$ I. Thiele ${ }^{3}$ B. Chimier, ${ }^{1}$ and G. Duchateau $\odot^{1, *}$ \\ ${ }^{1}$ University of Bordeaux-CNRS-CEA, Centre Lasers Intenses et Applications, UMR5107, 351 Cours de la Libération, F-33405 Talence, France \\ ${ }^{2}$ Faculty of Physics, L. V. Lomonosov Moscow State University, Moscow, Russia \\ ${ }^{3}$ Department of Physics, Chalmers University of Technology, SE-412 96 Göteborg, Sweden
}

(Received 25 November 2020; revised 18 February 2021; accepted 24 February 2021; published 8 March 2021)

\begin{abstract}
Controlling the electron dynamics during laser-matter interactions is a key factor to control the energy deposition and subsequent material modifications induced by femtosecond laser pulses. One way to achieve this goal is to use two-color femtosecond laser pulses. In this paper, the electron dynamics in dielectric materials induced by two-color femtosecond laser pulses is studied by solving dedicated optical Bloch equations. This model includes photo- and impact ionization, the laser heating of conduction electrons, their recombination to the valence band, and their collisions with phonons. The influence of photon energies, laser intensities, and pulse-to-pulse delay is analyzed. Depending on the interaction process, colors cooperate to excite electrons or drive them independently. For the given laser parameters, an optimal pulse-to-pulse delay is found which enhances significantly the energy deposition into the material, in agreement with experimental observations.
\end{abstract}

DOI: 10.1103/PhysRevA.103.033107

\section{INTRODUCTION}

The manufacturing industry widely uses micromachining (from the $\mu \mathrm{m}$ to $\mathrm{mm}$ scale) based on laser ablation [1-3] in microelectronics and many other high-tech fields. Motivated by the impressive advancement of laser technologies' ability to nowadays supply high-intensity ultrashort pulses, this technology has experienced a strong development in the last four decades [4] since it offers rapidity, versatility (different types of materials can be processed such as metals, plastics, ceramics, and glass), outstanding machining quality, and high precision (small features can be machined with little damage to the surrounding unirradiated regions of the material). A large number of applications have emerged including surface processing such as patterning [5-7], texturing [8-11], and etching $[12,13]$, and material removal such as cutting and drilling $[14,15]$. In these applications, the laser pulse interacting with the dielectric target first drives electrons into excited states through photo- and impact ionization together with laser heating in the conduction band, and their subsequent relaxation towards the lattice through collisions leads to an effective energy deposition into the material [16-20]. If this deposited energy exceeds locally a given threshold of the material, then irreversible macroscopic material modifications take place a few picoseconds later.

To further optimize such laser processes by tailoring the electron dynamics and energy deposition, pulse-shaping techniques consisting of complex temporal envelopes, frequency chirps, or a mixture of colors are developed [21-29]. The use of two-color laser pulses, i.e., two laser pulses with different colors, is of particular interest since specific processes of

\footnotetext{
*guillaume.duchateau@u-bordeaux.fr
}

electron dynamics depend on the photon energy [25-31]. A high-frequency pulse is efficient for seeding electrons through multiphoton ionization whereas a lower-frequency pulse is advantageous to heat these electrons and then cause material damage by an electron avalanche [3,32]. Since both wavelengths introduce opposite behaviors, by delaying in time the two laser pulses, one expects to find configurations that optimize the energy deposition into the material as it was observed in particular in Ref. [25].

To support those experimental developments in terms of understanding the physical mechanisms at play and predicting new experimental configurations, modeling approaches describing the laser-induced electron dynamics by femtosecond pulses in dielectric materials are required. Commonly used models devoted to evaluating the laser energy deposition rely on optical cycle-averaged electron transition rates assuming a monochromatic pulse [16-18,33,34]. Despite the latter approaches having provided interesting results, they are not well adapted to laser characteristics departing from the monochromatic case. For instance, for a frequency chirped pulse, the frequency is no longer well defined whereas it is required for the commonly used Keldysh expression for photoionization which assumes a monochromatic pulse [33,35]. In the case of the overlap of two pulses with different colors, the latter approach is not able to account for ionization pathways involving a mixture of both photon energies, i.e., it is only able to describe the photoionization induced independently by each color pulse. Other approaches, based on optical Bloch equations, to overcome the previous limitation have been introduced [35-37]. But to our knowledge they do not include collisional processes which are in particular responsible for impact ionization, which is a key process involved in the laser energy deposition into dielectric materials. In the present paper, we thus use our recently developed model based on 
optical Bloch equations, hereafter referred to as OBE [38], which is a time-dependent approach including direct interband transitions as well as collisional processes including impact ionization.

Within the framework of the enhancement of the laser energy deposition into dielectric materials by two-color laser pulses, this OBE model is used in the present paper to reach the following three objectives: (i) This study provides an indepth theoretical understanding of the mechanisms at play for the interaction of two-color pulses with a dielectric material based on an OBE model describing time-dependent electron transitions in the band structure directly induced by the laser field and through collisional processes. This electron dynamics includes photoionization, impact ionization, collisions with phonons, and recombination. (ii) To study such a two-color pulse configuration is of interest for application purposes since an optimization of the energy deposition seems possible [25,28,29]. Further advances on such studies can benefit from an in-depth understanding of the present interaction. This work thus can support the design of future experiments based on multiple colors. (iii) This work allows us to further theoretically validate the OBE model within conditions different from Ref. [38] by showing that this model correctly captures the simultaneous influence of both colors (including the mixture of both photon energies to photoionize). With laser parameters chosen to carry out previous studies, the experimental data of Ref. [25] further support the model validity since similar trends are predicted.

This paper is organized as follows. The OBE model is first introduced in Sec. II where in particular the model assumptions are recalled. In Sec. III, the single process of photoionization driven by two-color laser pulses is analyzed, independently of other processes. In Sec. IV, we study, also independently of other processes, the conduction electron heating driven by a laser field coupling infrared $(1.25 \mathrm{eV}$, $\approx 992 \mathrm{~nm})$ and ultraviolet $(2.5 \mathrm{eV}, \approx 496 \mathrm{~nm})$ colors with various intensity ratios. In Sec. $\mathrm{V}$, the full electron dynamics is studied, including also the impact ionization and recombination. In Sec. VI, a delay between the two colors is added in order to determine which configuration optimizes the laser energy deposition into the material. Conclusions are drawn in Sec. VII. For the reader's convenience, Appendixes provide some theoretical details, in particular revisiting the contribution of specific processes as described by optical Bloch equations.

\section{THEORETICAL DESCRIPTION}

The phenomenology of the femtosecond laser-induced electron dynamics in dielectric materials is as follows. First, the photoionization process consists of the promotion of valence electrons to the bottom of the conduction band (CB) through multiphoton absorption or tunneling. The produced conduction electrons further absorb photons, leading them to excited states with higher energies. Such an excitation is driven by two mechanisms: direct noncollisional transitions [39] or phonon-, ion-, or electron-assisted collisions $[34,40]$. Electron collisions with other particles, without involving photon absorption, also take place. That first leads to a change in the electron energy distribution. Second, diffusion processes induce a decoherence between the electron and the laser electric field due to a momentum exchange, which is hereafter referred to as coherence loss [41]. Conduction electrons also recombine to the valence band on a given timescale.

Such a laser-induced electron dynamics in dielectric materials can be described by optical Bloch equations designed to account for all those main interaction processes. A sketch of the model description is provided below, and more details can be found in Ref. [38].

The evolution of the density matrix $\hat{\rho}$ describing the electron system is governed by the OBE,

$$
\partial_{t} \hat{\rho}=\hat{\mathcal{L}}(\hat{\rho})+\hat{\mathcal{G}}(\hat{\rho}),
$$

where the energy dispersion relation along the Brillouin zone is not taken into consideration $[38,42] . \hat{\mathcal{L}}(\hat{\rho})$ is the Liouvillevon Neumann operator [43],

$$
\hat{\mathcal{L}}(\hat{\rho})=-\frac{i}{\hbar}[\hat{H}, \hat{\rho}]=-\frac{i}{\hbar}(\hat{H} \hat{\rho}-\hat{\rho} \hat{H}),
$$

where $\hbar$ is the reduced Planck constant, and $\hat{\mathcal{G}}(\hat{\rho})$ is an operator that introduces the impact ionization, collisions, and recombination. In Eq. (2), $\hat{H}$ is the Hamiltonian of the electron subsystem, which is real and symmetric $\left(\hat{H}=\hat{H}^{T}\right)$. It is modeled as the sum of the unperturbed Hamiltonian $\hat{H}_{0}$, which contains the energy levels of the band structure, and the interaction Hamiltonian $\hat{H}_{\text {int }}$, accounting for the laser interaction with the electron system,

$$
\hat{H}=\hat{H}_{0}+\hat{H}_{\text {int }} \text {. }
$$

The unperturbed Hamiltonian in Eq. (3) is a diagonal matrix including all the energy levels. The interaction Hamiltonian in Eq. (3) is calculated with the dipole approximation in the length gauge, i.e., $\hat{H}_{\text {int }}=-e E(t) \hat{\mu}$. In the latter expression, $e$ is the elementary charge, $E(t)$ is the time-dependent laser electric field, and $\hat{\mu}$ is the dipole-transition matrix, which is real and symmetric. The input parameters of this model, the energy levels, electric field, and dipole-transition matrix are provided below.

The operator $\hat{\mathcal{G}}(\hat{\rho})$ introduces in Eq. (1) the impact ionization $\hat{\mathcal{G}}_{\text {imp }}$, the coherence loss between levels due to collisions $\hat{\mathcal{G}}_{\text {coh }}$, and the recombination $\hat{\mathcal{G}}_{\text {rec }}$ :

$$
\hat{\mathcal{G}}(\hat{\rho})=\hat{\mathcal{G}}_{\text {rec }}(\hat{\rho})+\hat{\mathcal{G}}_{\text {imp }}(\hat{\rho})+\hat{\mathcal{G}}_{\text {coh }}(\hat{\rho})
$$

$\hat{\mathcal{G}}$ is built to satisfy the properties of the density matrix recalled in Appendix A [44]. Briefly, the impact ionization model bridges the electron in the highest $\mathrm{CB}$ level to the electron in the valence state, resulting in two electrons in the lower-energy level of the conduction band, with a characteristic timescale $\tau_{\text {imp. }}$. The coherence loss is modeled by an exponential decay of the off-diagonal elements of the density matrix with a characteristic timescale $\tau_{\text {coh }}$. The recombination is described by an exponential decay of the diagonal elements of the density matrix with a characteristic timescale $\tau_{\text {rec }}$.

Following this description, the state of the electron system is completely defined by the density matrix $\hat{\rho}$ [45]. The on-diagonal element $\rho_{j, j}$ is always real and represents the probability of finding the electron in the $j$ th energy level (thus $0 \leqslant \rho_{j, j} \leqslant 1$ ). The off-diagonal element $\rho_{j, k}$, with $j \neq k$, is 
complex: Its module represents the probability for a transition between the $j$ th and $k$ th energy levels, and its argument corresponds to the phase of Rabi oscillations between those states (see Appendix B).

The dielectric material is characterized by a band structure containing $N+1$ energy levels. The lowest-energy level (labeled with index $j=0$ ) represents the valence band (VB) and the rest of the energy levels $(1 \leqslant j \leqslant N)$ belong to the conduction band (CB). Without loss of generality, the energy of the first CB level $(j=1)$ is set to zero $\left(E_{1}=0\right)$ and, hence, $E_{0}=-E_{g}$, where $E_{g}$ is the gap energy of the dielectric material. Moreover, the highest $\mathrm{CB}$ level that we consider, namely, $j=N$, must have an energy $E_{N}-E_{1}$ that is sufficiently large to fulfill energy and momentum conservation during the impact ionization process [34,46,47]: $E_{N}-E_{1} \geqslant 3 E_{g} / 2$. The $\mathrm{CB}$ energy levels are equally spaced [48] by the minimum photon energy in case of two-color pulses, $E_{j}-E_{1}=(j-1) \hbar \omega$, to ensure resonant conditions [38]. The number of levels is thus $N=1+\left\lfloor\frac{1.5 E_{g}}{\hbar \omega}\right\rfloor$, where $\lfloor x\rfloor$ is the floor function (a maximum integer that is less than or equal to $x$ ).

Assuming the material is noncentrosymmetric, the elements of the dipole-transition matrix $\hat{\mu}$ are constructed as follows. First, $\mu_{0,0}$ has a nonzero value (this means that the VB does not have a well-defined parity) and $\mu_{0,1}=$ $\mu_{1,0}$. Then, the transition matrix elements for the transitions between the VB level and the rest of CB levels $(j>$ 1) are $\mu_{0, j}=\mu_{j, 0}=\mu_{0,1}\left(E_{1}-E_{0}\right) /\left(E_{j}-E_{0}\right)$, corresponding to ionization and above-threshold ionization processes. The transitions between CB levels, $\mu_{j, k}$ with $j>0$ and $k>0$, which represent the laser-induced electron heating process, are $\mu_{j, k}=\mu_{k, j}=C_{\mu} /\left|E_{k}-E_{j}\right|$, where $C_{\mu}$ is a coefficient which is set so that the conductivity of $\mathrm{CB}$ electrons is of the appropriate order of magnitude (see Appendix C). All conduction levels are connected to each other through the dipole-transition matrix, ensuring that photons with different energies can be absorbed on equal footing in the heating process. Despite that $\mu_{j, j}$ should be different from zero since all levels are connected (i.e., they do not exhibit a defined parity), we set $\mu_{j, j}=0$ for $j \geqslant 1$ to avoid additional free parameters, without loss of generality to model the present physical system.

As shown below, correct trends are obtained which validate a posteriori the above-mentioned modeling assumptions. We recall as mentioned in Ref. [38] that the present OBE model includes assumptions since we proceed with a step-by-step approach to build such a modeling. The present model version allows us to clearly interpret the predicted results and highlight the main physical processes at play by capturing the main behaviors of the laser-induced electron dynamics in dielectric materials. Note that the proposed OBE model was validated in Ref. [38] against the state-of-the-art multiple rate equations in conditions where the latter approach is valid (for a long enough pulse where the transition rates based on optical cycle averages are valid). Within the same approach, the collision frequencies are chosen to be a constant with a standard order of magnitude. As detailed below, similar results are obtained by varying these values in a reasonable range. For application purposes of this model, also note that it is computationally as efficient as multiple rate equations [34] owing to the development of an advanced resolution algorithm
[38]. A standard run takes a few seconds of CPU time with one core.

The two-color laser electric field is described as

$$
E(t)=\sum_{l=1}^{2} E^{(l)}(t),
$$

with

$$
E^{(l)}(t)=a_{l} \sin ^{2}\left[\pi\left(t-t_{l}\right) / \tau_{l}\right] \sin \left[\omega_{l}\left(t-t_{l}\right)+\phi_{l}\right],
$$

where $E_{l}(t)$ is characterized by its wavelength $\lambda_{l}=2 \pi c / \omega_{l}(c$ is the speed of light), its amplitude $a_{l}$, and its phase $\phi_{l}$. Each pulse is defined in the time interval $\left[t_{l}, t_{l}+\tau_{l}\right]$. Its duration $\tau_{l}$ is assumed to be equal to an integer number of cycles [i.e., $\tau_{l} /\left(2 \pi / \omega_{l}\right)$ is an integer], which ensures that the integral of the laser color over the whole time interval is zero. The time shift between the two colors is defined as $\Delta t_{1,2}=t_{2}-t_{1}$. Throughout this paper, without loss of generality, we consider $\omega_{2}>\omega_{1}$ and $t_{1}=0$.

In the present paper, we are mainly interested in two observable values: the ionization degree and the electron energy density. The expectation value of the ionization degree is given by the sum of the probabilities of finding electrons in conduction levels,

$$
Z(t)=\sum_{j>0} \rho_{j, j}(t),
$$

satisfying $0 \leqslant Z \leqslant 1$. The laser-induced electron heating is characterized by the energy density of excited conduction electrons,

$$
U_{\mathrm{CB}}(t)=\sum_{j>0} \mathcal{N}_{0}\left(E_{j}-E_{1}\right) \rho_{j, j}(t)
$$

where $\mathcal{N}_{0}$ is the density of neutral atoms of the dielectric material.

For simulations, we consider that the parameters of the dielectric target are close to those of the fused silica which is a widely studied material. We thus use a gap energy of $E_{g}=7.5 \mathrm{eV}$ [32] and a density of neutral atoms of $\mathcal{N}_{0}=$ $2.2 \times 10^{22} \mathrm{~cm}^{-3}$ [49]. For the transition dipole matrix, we take $\mu_{0,0}=2.0 \AA, \mu_{0,1}=\mu_{1,0}=0.5 \AA$, and $C_{\mu}=0.45 \mathrm{eV} \AA$. Moreover, we consider $\tau_{\text {rec }}=150 \mathrm{fs}$ [50-52], $\tau_{\text {imp }}=1 \mathrm{fs}$, and $\tau_{\text {coh }}=10$ fs for coherence relaxation driven by electronphonon collisions [42,49,53,54].

The two laser pulses have a sufficiently long duration $\tau_{1}=$ $\tau_{2} \approx 300 \mathrm{fs}$ in order to have a narrow spectrum around $\omega_{1}$ and $\omega_{2}$ within the present range of photon energies (the number of optical cycles is of the order of 100). Doing so allows us to simplify the analysis of forthcoming results including the driven electron dynamics by the mixture of photon energies.

There is no delay $\left(\Delta t_{1,2}=0\right)$ and no phase shifts $\left(\phi_{1}=\right.$ $\phi_{2}=0$ ), except for the study on the influence of the delay between two pulses (Sec. VI). Within these conditions, both electric fields can be characterized by the total intensity $I=$ $n_{0} c \epsilon_{0}\left(a_{1}^{2}+a_{2}^{2}\right) / 2$, where $\varepsilon_{0}$ is the vacuum permittivity, $n_{0}=$ 1.5 is the material refractive index, and we define the color-tocolor intensity ratio $r=a_{2}^{2} /\left(a_{1}^{2}+a_{2}^{2}\right)$. The total intensity will be varied from $10^{11} \mathrm{~W} / \mathrm{cm}^{2}$ up to $10^{14} \mathrm{~W} / \mathrm{cm}^{2}$, and the ratio $r$ is varied from 0 (pure $\omega_{1}$ color) to 1 (pure $\omega_{2}$ color). 

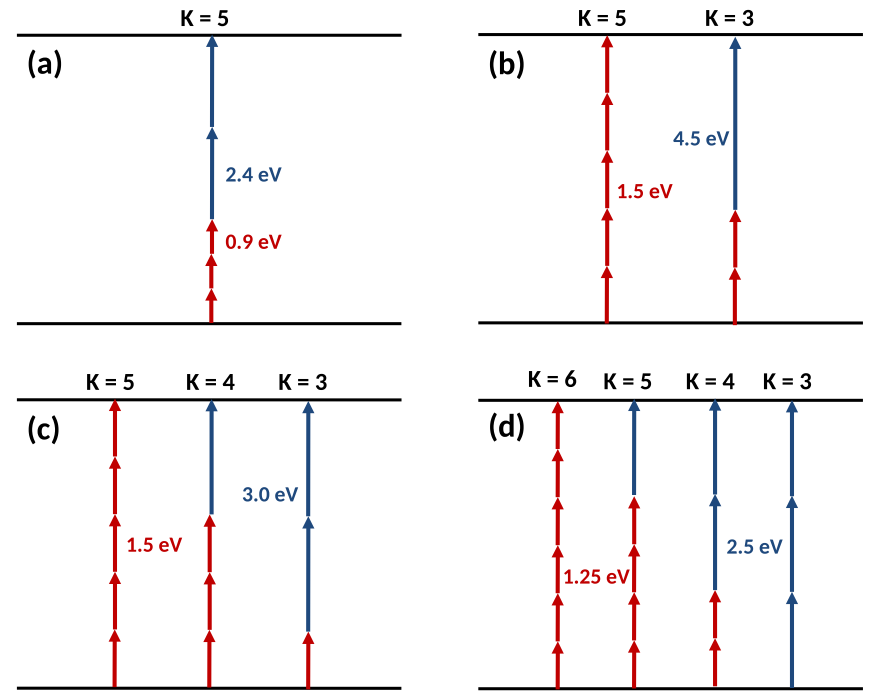

FIG. 1. Resonant ionization paths made of $\hbar \omega_{1}$ photons (red shortest arrow) and $\hbar \omega_{2}$ photons (blue longest arrow). The energy gap is $E_{g}=7.5 \mathrm{eV}$. (a) Two incommensurable photon energies with a unique five-photon resonant combination. (b) Two commensurable photon energies with five- and three-photon resonant combinations. (c) Two commensurable photon energies with five-, four-, and three-photon resonant combinations. (d) Two commensurable photon energies with six-, five-, four-, and three-photon resonant combinations.

\section{TWO-COLOR LASER-INDUCED PHOTOIONIZATION}

The photoionization driven by the two-color laser field [Eq. (5)] is addressed in this section. Only the levels $j=0$ and $j=1$ in OBE are included, and the operator $\hat{\mathcal{G}}(\hat{\rho})$ is removed (i.e., no collisional process is taken into account). The initial condition is $\rho_{1,1}=0$ and $\|\hat{\rho}\|=1$ (see Appendix A). The photon energies are chosen so that their combinations bridge resonantly the band gap in order to simplify the analysis of the forthcoming results (see Fig. 1), i.e., $E_{g}=n_{1} \hbar \omega_{1}+n_{2} \hbar \omega_{2}$ with $n_{1}$ and $n_{2}$ integers. Three characteristic situations are chosen in order to highlight various ways of cooperation of colors to ionize: (A) $\hbar \omega_{2}$ is not a multiple of $\hbar \omega_{1}$, i.e., $\hbar \omega_{1}=$ $0.9 \mathrm{eV}$ and $\hbar \omega_{2}=2.4 \mathrm{eV}$; (B), (C) $\hbar \omega_{2}$ is a multiple of $\hbar \omega_{1}$, and only the pure-color 1 can bridge resonantly the band gap (pure-color 2 cannot), i.e., $\hbar \omega_{1}=1.5 \mathrm{eV}$ and $\hbar \omega_{2}=4.5 \mathrm{eV}$, and $\hbar \omega_{1}=1.5 \mathrm{eV}$ and $\hbar \omega_{2}=3$. eV; (D) $\hbar \omega_{2}$ is a multiple of $\hbar \omega_{1}$, and both pure colors can bridge resonantly the band gap, i.e., $\hbar \omega_{1}=1.25 \mathrm{eV}$ and $\hbar \omega_{2}=2.5 \mathrm{eV}$. The number of absorbed photons is $K=n_{1}+n_{2}$. The ionization is expected to behave as $I^{K}$ under perturbative conditions [55,56]. Various photoionization paths are possible depending on the combination of photon energies as shown in Fig. 1 (for a given number of photons $K$, various permutations are possible but only one is shown). Going from cases (a) to (d), the number of allowed quantum pathways increases from 1 to 4 .

Figure 2 shows the evolution of the ionization degree $Z$ at the end of pulses with respect to the above-defined laser parameters. Figures 2(a) and 2(d) show the evolution of $Z$ with respect to both $I$ and $r$ for cases $\mathrm{A}$ and $\mathrm{D}$, respectively. The behaviors for both cases $\mathrm{B}$ and $\mathrm{C}$ are similar to case A (not shown). Two main characteristics appear: (i) For a given $r$, for all cases, $Z$ first increases monotonically as a function of $I$, and then exhibits an oscillationlike behavior; (ii) for a given $I$, cases A, B, and C exhibit a symmetric structure whereas $Z$ increases monotonically as a function of $r$ for case D.

To better depict the behaviors with respect to $I$, sections of the previous color map are provided by Figs. 2(b), 2(c), 2(e), and 2(f) for cases A-D, respectively. For all parameters, the general behavior is similar to the well-known singlecolor case $[35,38]$. The monotonic increase corresponds to the perturbative regime of multiphoton absorption (up to intensities of the order of $10^{13} \mathrm{~W} / \mathrm{cm}^{2}$ within the present conditions) where we have checked that the slopes [estimated
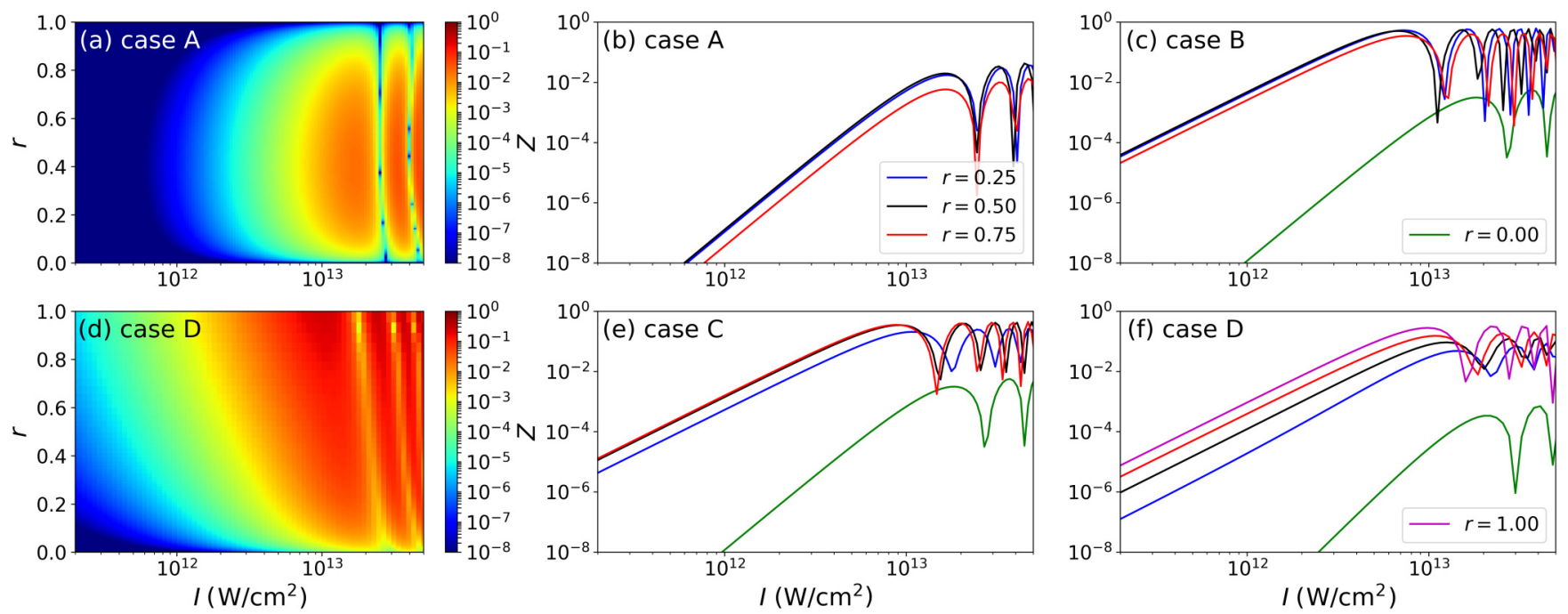

FIG. 2. Ionization degree given by the two-level Bloch model at the end of the laser pulse as a function of $I$ and $r$ : (a) case of Fig. 1(a) and (d) case of Fig. 1(d). Ionization degree as a function of $I$ and for several values of $r$ (same legend for these four subplots): (b) case of Fig. 1(a), (c) case of Fig. 1(b), (e) case of Fig. 1(c), and (f) case of Fig. 1(d). For the printed grayscale version, the following information allows one to distinguish different curves. (b), (c), (e), (f) The lines from the top to bottom at $I=10^{12} \mathrm{~W} / \mathrm{cm}^{2}$ correspond to $r=1, r=0.75, r=0.5$, $r=0.25$, and $r=0$, respectively. 

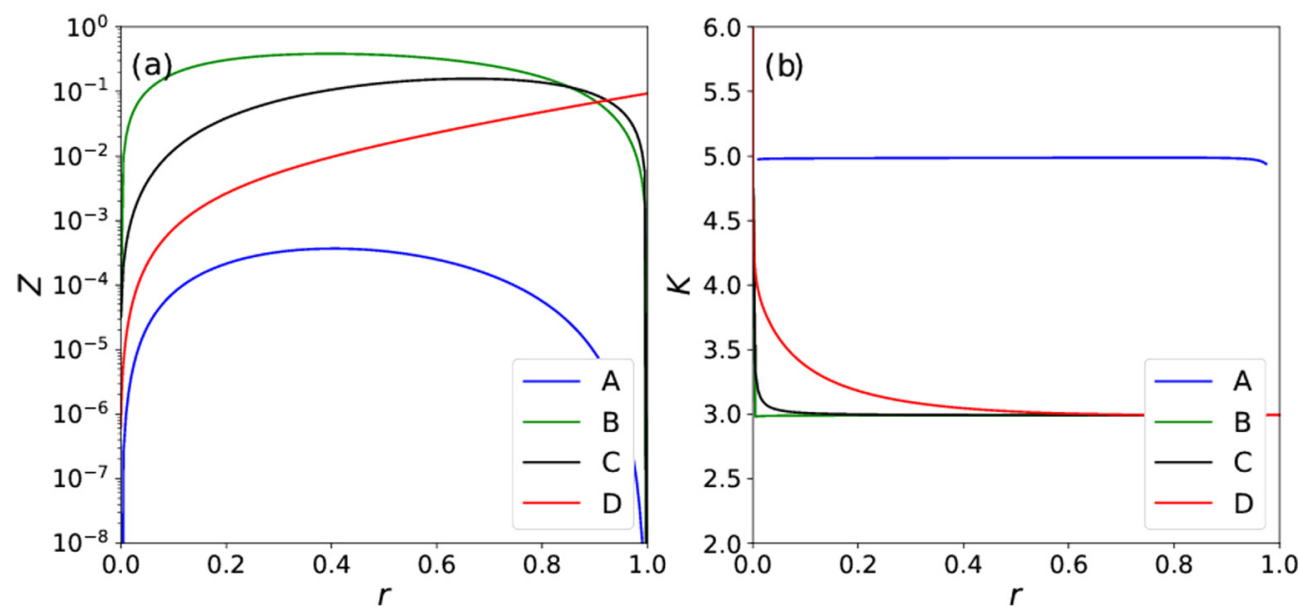

FIG. 3. (a) Ionization degree as a function of $r$ at $I=5 \times 10^{11} \mathrm{~W} / \mathrm{cm}^{2}$, for the cases of Fig. 1 (see legend). (b) Number of absorbed photons as a function of $r$ at $I=5 \times 10^{11} \mathrm{~W} / \mathrm{cm}^{2}$, for the cases of Fig. 1 (see legend).

as $\approx d(\ln Z) / d(\ln I)]$ are the same as the expected values of $K$ depending on the parameters (Fig. 1). For instance, $K=5$, i.e., $Z \propto I^{5}$, for case A [Fig. 2(b)]. For intensities above $\sim 10^{13}$ $\mathrm{W} / \mathrm{cm}^{2}, Z$ exhibits an oscillationlike behavior accounting for reaching nonperturbative conditions where tunneling ionization contributes significantly [35,57].

The main differences between cases are observed when $r$ is changed. Going from cases A to D, the curves evolve more with respect to $r$. Depending on the case, $K$ evolves differently with respect to $r$. Figure 3 quantifies these observed trends for $I=5 \times 10^{11} \mathrm{~W} / \mathrm{cm}^{2}$. Figure 3(a) shows the evolution of $Z$ with respect to $r$. The evolution of $Z$ for case $\mathrm{D}$ is clearly different from those of cases $\mathrm{A}-\mathrm{C}$, the latter exhibiting a roughly symmetric shape. This is due to the fact that for a pure-color pulse, the $\omega_{1}$ pulse involves a large $K$ (ionization probability is thus small) and the $\omega_{2}$ pulse does not bridge the band gap, thus also leading to a low $Z$. A maximum value of $Z$ is then obtained for a mixture of both colors. In case D, the $\omega_{2}$ pulse does bridge the band gap with a low multiphoton order, thus giving rise to high $Z$, explaining the monotonic increase with respect to $r$. The evolution of $K$ as a function of $r$ is shown in Fig. 3(b). It confirms that case D provides the largest evolution of $K$ with respect to $r$, accounting for the largest number of allowed ionization pathways. In all cases, comparing to Fig. 1, it is confirmed that the present OBE model well accounts for the cooperation of pulses with different colors to the ionization process. Note that the previous conclusions remain similar for another intensity where the multiphoton regime still stands.

Since case D exhibits the largest sensitivity with respect to the color amplitude ratio, only this case is considered hereafter.

As a final comment for this section, note that the present OBE model still stands when the ionization degree reaches unity, inducing a strong energy absorption. For femtosecond laser pulses, the lattice does not have enough time to evolve significantly during the interaction. Material decomposition takes place on a longer timescale, at least a few picoseconds, where the electron energy transfer to the lattice is significant $[58,59]$. Then the increase in the lattice temperature can indeed induce a phase transition and material decomposition for a large ionization degree for which the laser energy absorption is significant. It follows that within the present conditions where the material structure has no time to evolve, the assumption of constant electron energy levels is reliable. With the photon energies used in the present work, the critical density is of the order of a few $10^{21} \mathrm{~cm}^{-3}$ which roughly corresponds to $Z=0.1$. In that case we observe (see below) that the associated energy density is of the order of a few $\mathrm{kJ} / \mathrm{cm}^{3}$ which consistently corresponds to the criterion for material modifications. For $Z>0.1$, laser propagation is strongly affected and may modify the present results so that the present conclusions only make sense on the material surface where the incident laser intensity is not modified by the material response.

\section{TWO-COLOR LASER-INDUCED ELECTRON HEATING}

In this section, only the electron heating process (only transitions in the conduction band) driven by the two-color laser field is studied. Photon energies are those of case D [Fig. 1(d)]: $\hbar \omega_{1}=1.25 \mathrm{eV}$ and $\hbar \omega_{2}=2.5 \mathrm{eV}$. The considered system is described as follows. The valence-band level $(j=0)$ is removed by switching off the ionization processes. The conduction band is described with $N$ states, where the index goes from $j=1$ to $j=N$. To account for conditions of the next section where impact ionization is allowed, we set $N=11$. Only the Liouville-von Neumann and the decoherence $\hat{\mathcal{G}}_{\text {coh }}(\hat{\rho})$ operators are used. The former induces electron heating, and the latter accounts for collisions inducing coherence loss. The initial condition is $\rho_{1,1}=1$ and $\|\hat{\rho}\|=1$ (see Appendix A).

Figure 4(a) shows the energy density gained by conduction electrons as a function of $I$ and $r$, at the end of the laser pulse (i.e., at $t=\tau_{1}=\tau_{2}$ ), for $\tau_{\text {coh }}=10$ fs. Both colors are able to heat the electrons but, as expected, infrared is more efficient than ultraviolet. In Fig. 4(b) we can observe that, for not too high intensities, the energy gained by $\mathrm{CB}$ electrons is proportional to the laser intensity [46,60,61], i.e., $U_{\mathrm{CB}} \propto I$ independently of the value of $r$. This is in agreement with the Drude model and, in Appendix C, we verify that the value of the corresponding conductivity of $\mathrm{CB}$ electrons in 

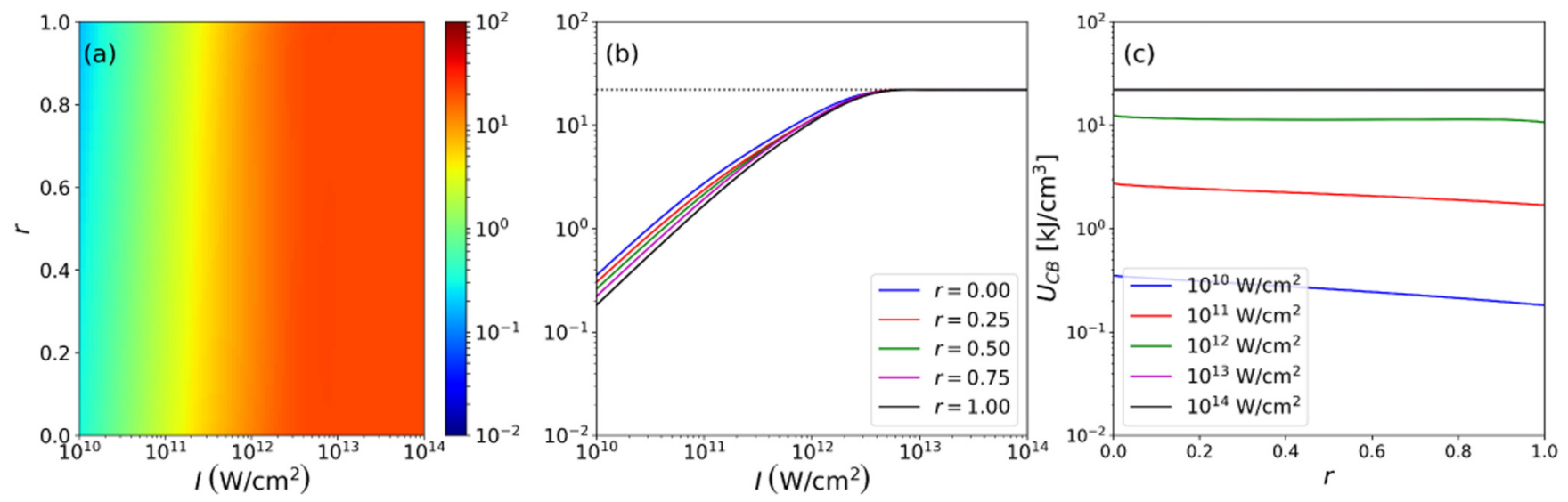

FIG. 4. Energy density gained by CB electrons, at the end of the laser pulse, for the two-color laser of Fig. $1(\mathrm{~d})$, with $\tau_{\text {coh }}=10 \mathrm{fs}$. (a) $U_{\mathrm{CB}}$ as a function of $I$ and $r$. (b) $U_{\mathrm{CB}}$ as a function of $I$ and several values of $r$. The horizontal dotted line represents the energy density at the equilibrium state given by Eq. (9), towards which $U_{\mathrm{CB}}$ saturates at high intensities in the presence of collisions. (c) $U_{\mathrm{CB}}$ as a function of $r$ and several values of $I$. For the printed grayscale version, the following information allows one to distinguish different curves. (b) The lines from the bottom to top at $I=10^{10} \mathrm{~W} / \mathrm{cm}^{2}$ correspond to $r=1, r=0.75, r=0.5, r=0.25$, and $r=0$, respectively. (c) The lines from the bottom to top for all $r$ correspond to $I=10^{10} \mathrm{~W} / \mathrm{cm}^{2}, I=10^{11} \mathrm{~W} / \mathrm{cm}^{2}, I=10^{12} \mathrm{~W} / \mathrm{cm}^{2}, I=10^{13} \mathrm{~W} / \mathrm{cm}^{2}$, and $I=10^{14} \mathrm{~W} / \mathrm{cm}^{2}$, respectively.

our Bloch model is compatible with the values of fused silica. Precisely because of this proportionality to the laser intensities, in Fig. 4(c) we can see that the profiles are linear with $r$ for sufficiently low laser intensities.

For higher intensities, Fig. 4(b) shows that the energy density saturates. If all electrons are promoted to the highest states, the maximum energy density is $44 \mathrm{~kJ} / \mathrm{cm}^{3}$ with the present parameters. Actually, as explained in Appendix D, collisions lead the electron system to an equilibrium state such that $\rho_{1,1}=\cdots=\rho_{N, N}=1 / N$. According to Eq. (8), the energy density gained by the electrons at this equilibrium state is

$$
U_{\mathrm{CB}, \text { equilibrium }}=\mathcal{N}_{0} \frac{E_{N}-E_{1}}{2} \approx 22 \mathrm{~kJ} / \mathrm{cm}^{3},
$$

which is plotted in Fig. 4(b) as the horizontal dotted black line which indeed appears as the maximum energy density. It is worth noting that this maximum value is significantly larger than the laser-induced modification threshold of fused silica, whose value is of the order of $4 \mathrm{~kJ} / \mathrm{cm}^{3}$ by considering the melting temperature (softening point), $T_{\text {melt }} \approx 1500^{\circ} \mathrm{C}$ $[32,62]$, as a modification criterion. Thus the observed saturation in the absorbed energy density does not represent any limitation to study the energy deposition into the material. For other application purposes requiring a higher-energy deposition, $N$ can be increased, thus increasing the absorbed energy limit.

In order to study the influence of collisions through the decoherence time, the evolution of the absorbed energy density as a function of the total laser intensity is shown in Fig. 5 for $\tau_{\mathrm{coh}}=1 \mathrm{fs}$ (strong collisions), $\tau_{\mathrm{coh}}=10 \mathrm{fs}$, and $\tau_{\mathrm{coh}} \rightarrow \infty$ (no collisions). Various values of $r$ are also used which all lead to the same expected behavior: The longer the collisional decoherence time, the larger is the electron energy density. Indeed, the collisions break the coherence allowing electrons to efficiently transit sequentially through conduction states to reach higher energies. Note that coherence loss plays a role for both up and down transitions. But since the electron population is larger for lower levels, it mainly affects the transitions toward higher states. This resonant nature of the electron dynamics in the conduction band is highlighted by the oscillatinglike behavior of the energy density for sufficiently high intensities when there is no collision. Note that a collision time in the fs range provides an absorbed energy density of a few $\mathrm{kJ} / \mathrm{cm}^{3}$ for threshold intensities of

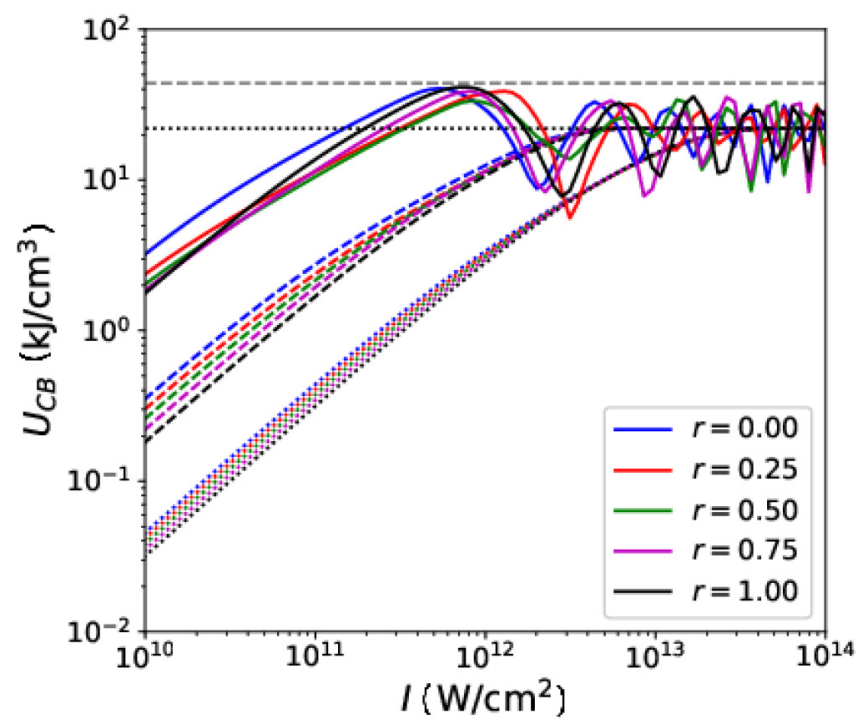

FIG. 5. $U_{\mathrm{CB}}$ as a function of $I$ and several values of $r$ : without collisions (solid lines), with weak collisions $\tau_{\mathrm{coh}}=10 \mathrm{fs}$ (dashed lines), and with strong collisions $\tau_{\mathrm{coh}}=1$ fs [dotted lines, which are the same curves plotted in Fig. 4(b)]. The horizontal dotted line in black represents the energy density of Eq. (9). The maximum energy that the electron subsystem can achieve is depicted by the horizontal dashed line in gray. For the printed grayscale version, the following information allows one to distinguish different curves. For each bunch of curves, the lines from the bottom to top at $I=$ $10^{10} \mathrm{~W} / \mathrm{cm}^{2}$ correspond to $r=1, r=0.75, r=0.5, r=0.25$, and $r=0$, respectively. 


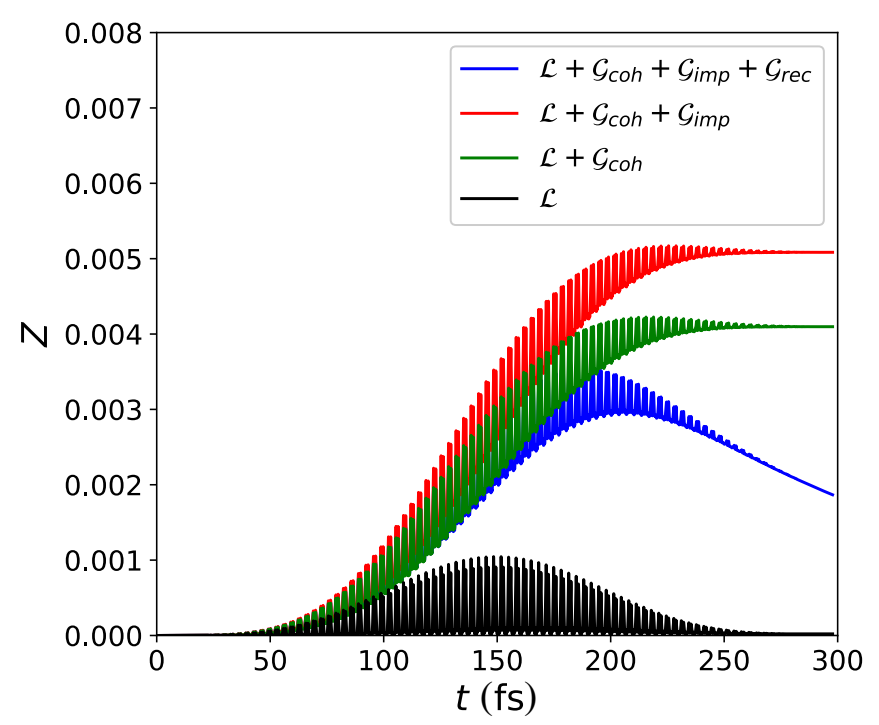

FIG. 6. Time evolution of the ionization degree $Z$ induced by the two-color laser of Fig. 1(d) with $I=10^{12} \mathrm{~W} / \mathrm{cm}^{2}$ and $r=0.5$, calculated according to different right-hand-side terms in Eq. (1). The simulation starts at $t_{1}=t_{2}=0$ fs. For the printed grayscale version, the following information allows one to distinguish different curves. The lines from the bottom to top at $t=300 \mathrm{fs}$ correspond to the model version $\mathcal{L}, \mathcal{L}+\mathcal{G}_{\text {coh }}+\mathcal{G}_{\text {imp }}+\mathcal{G}_{\text {rec }}, \mathcal{L}+\mathcal{G}_{\text {coh }}$, and $\mathcal{L}+$ $\mathcal{G}_{\text {coh }}+\mathcal{G}_{\text {imp }}$, respectively.

the order of $10 \mathrm{TW} / \mathrm{cm}^{2}$, which corresponds to observed trends [32].

\section{FULL ELECTRON DYNAMICS INDUCED BY TWO-COLOR NOT-DELAYED LASER PULSES}

The whole OBE model is considered here, with all operators and a full band structure (energy levels from $j=0$ to $j=N$ ), to study the contributions of photoionization, impact ionization, electron heating, and recombination on the electron dynamics driven by a two-color laser field. The used parameters pertaining to fused silica are as follows: $\tau_{\text {rec }}=$ 150 fs [50-52], $\tau_{\text {imp }}=1$ fs, and $\tau_{\text {coh }}=\tau_{\text {imp }}$ for electronelectron collisions (i.e., the transitions between $j=N$ and the rest of the levels), $\tau_{\text {coh }} \rightarrow \infty$ for the coupling between $j=0$ and $j=1$ levels (because electrons at level $j=1$ are still bounded to their parent ion and thus they do not collide with phonons), and $\tau_{\mathrm{coh}}=10$ fs for electron-phonon collisions (involving the rest of the transitions between levels). The initial condition is $\rho_{0,0}=1$ and $\|\hat{\rho}\|=1$. The photon energies of case D [Fig. 1(d)] are still used: $\hbar \omega_{1}=1.25 \mathrm{eV}$ and $\hbar \omega_{2}=2.5 \mathrm{eV}$.

The temporal evolution of the electron populations is first considered. Figure 6 shows the ionization degree $Z$ with respect to time for $I=10^{12} \mathrm{~W} / \mathrm{cm}^{2}$ and $r=0.5$. Four calculations have been performed to exhibit the influence of various processes: Only the Liouville-von Neumann operator is included, the same description with the coherence-loss process in addition, the same model with the impact ionization in addition, and the full electron dynamics including also the recombination. The observed behaviors are similar to those obtained in the case of a single-color pulse [38].
The ionization dynamics only described by the Liouville-von Neumann operator is strongly reversible [35]. The latter is broken by the coherence loss leading to a more efficient freeelectron production. As expected, impact ionization further increases the ionization degree, whereas the recombination process has the opposite influence.

Figure 7 depicts the evolution of the ionization degree as a function of the total laser intensity $I$. Three color-tocolor intensity ratios $r=0 ; 0.5 ; 1$ are considered to show the pure-color dynamics and the cooperative configuration. As previously, four calculations have been performed to exhibit the influence of each process (the color legend is the same as in Fig. 6). When solely the Liouville-von Neumann operator is included, for not too high intensities for which the ionization through the multiphoton absorption regime stands $(I<$ $10^{13} \mathrm{~W} / \mathrm{cm}^{2}$ ), the expected multiphoton orders are found, i.e., $Z \propto I^{6}$ for IR and $Z \propto I^{3}$ for $\mathrm{UV}$, the latter generating more conduction electrons. When a mixture of both colors is considered, the multiphoton order is slightly larger than 3 , in agreement with the results of Sec. III.

The previous scaling together with the amplitude of $Z$ are strongly affected when the decoherence is introduced. Variations in amplitude have been discussed previously. The multiphoton order now is of the order of unity, i.e., $Z \propto I$. This behavior is observed for each conduction level $\left(\rho_{j, j} \propto I\right.$ for $1<j<N$ ) except for the first state $j=1$ which exhibits a scaling exponent closer $\left(\rho_{1,1} \propto I^{4}\right.$ for IR, and $\rho_{1,1} \propto I^{2}$ for UV) to the one arising from pure multiphoton absorption (without decoherence, results not shown). Collisions break the electron coherence with the laser electric field, and part of the electrons thus does not go back and forth on the valence and first $\mathrm{CB}$ states during the whole pulse duration, i.e., they are promoted irreversibly to the conduction band on the decoherence timescale. The associated number of optical cycles during this period is thus small, even less than one, and the photon energy is no longer well defined. The electron dynamics then exhibits a classical nature [63] in the sense where the electron absorbs the classical energy of the electric field $\left(\propto\left[\int E(t) d t\right]^{2}\right)$ which scales linearly with the intensity. Within the present OBE model, for $I \leqslant 10 \mathrm{TW} / \mathrm{cm}^{2}, Z$ is overestimated due to the above-described process which do not account for time-dependent dephasing rates [64]. However, with the present framework of material modifications where we are interested in a large absorbed laser energy leading to an electron energy density of a least a few $\mathrm{kJ} / \mathrm{cm}^{3}$, this regime does not take place. For $I \geqslant 10 \mathrm{TW} / \mathrm{cm}^{2}$, the expected slope is retrieved as also shown in Ref. [64]. For application purposes requiring lower intensities, the present OBE model could be improved with the ingredients of Ref. [64] but it is out of the scope of the present work.

The impact ionization also influences significantly the production of conduction electrons for intensities $>10^{13} \mathrm{~W} / \mathrm{cm}^{2}$. The longer the wavelength, the larger is the relative increase of $Z$ induced by impact ionization [25,38]. However, in absolute terms, since electrons must be first seeded from VB to $\mathrm{CB}$ through photoionization before contributing to the impact ionization process, the largest ionization degrees are obtained for the UV pulse. Finally, the recombination process decreases the value of $Z$ by a constant factor on the whole intensity range for all color configurations as expected. 

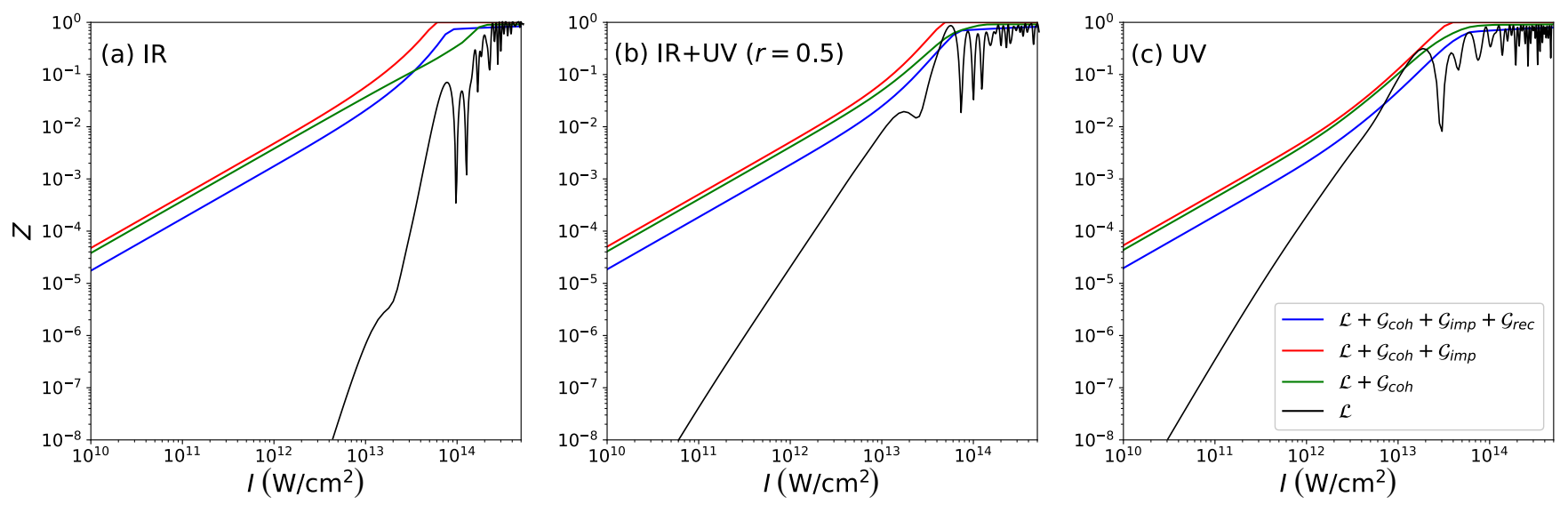

FIG. 7. Ionization degree $Z$ as a function of the overall intensity $I$ of the two-color laser of Fig. 1(d), calculated according to different right-hand-side terms in Eq. (1) and several values of $r$ : (a) $r=0$ (pure infrared), (b) $r=0.5$, and (c) $r=1$ (pure ultraviolet). For the printed grayscale version, the following information allows one to distinguish different curves. (a)-(c) The lines from the bottom to top at $I=10^{11}$ $\mathrm{W} / \mathrm{cm}^{2}$ correspond to the model version $\mathcal{L}, \mathcal{L}+\mathcal{G}_{\text {coh }}+\mathcal{G}_{\text {imp }}+\mathcal{G}_{\text {rec }}, \mathcal{L}+\mathcal{G}_{\text {coh }}$, and $\mathcal{L}+\mathcal{G}_{\text {coh }}+\mathcal{G}_{\text {imp }}$, respectively.

Figure 8 shows the energy density gained by $\mathrm{CB}$ electrons within the same conditions and legends as Fig. 7. The results exhibit significant differences with respect to Sec. IV where the $\mathrm{CB}$ was assumed to be initially filled (ionization processes were not included), leading to a linear evolution of $U_{\mathrm{CB}}$ with respect to the intensity. When only the Liouville-von Neumann operator is included, due to the nonlinear photoionization process, $U_{\mathrm{CB}}$ depends nonlinearly on $I$, with an exponent going from 2 for UV to 4 for IR (i.e., a balance between $\propto I^{K}$ of photoionization and $\propto I$ of the Drude model). Since the UV pulse leads to a significantly larger $Z$ than the IR pulse for a given intensity, $U_{\mathrm{CB}}$ is also larger for the UV pulse. The mixed-color case provides an intermediate result. When collisional decoherence is further included, $U_{\mathrm{CB}}$ increases compared to the previous case because of the rise in photoionization as discussed previously, and also now scales linearly as a function of the intensity. The increase is more pronounced for IR than UV because the former color is more efficient to heat. However, the UV provides a larger $U_{\mathrm{CB}}$ because more electrons are photoionized. For intensities $>10^{13} \mathrm{~W} / \mathrm{cm}^{2}$, we also see that impact ionization increases the electron heating more efficiently for IR than UV, until the Bloch system saturates at the limit discussed previously. Eventually, Fig. 8 shows that the recombination process diminishes the total energy gained by the $\mathrm{CB}$ electron for all laser intensities and approximately in the same factor. Overall, all previous trends account for expected behaviors.

\section{CONTROLLING THE ELECTRON DYNAMICS BY DELAYING THE TWO COLORS}

A time delay $\Delta t_{1,2}$ between each laser pulse is now introduced to study its influence on the ionization and the energy absorption. The photon energies of case $\mathrm{D}$ are always used [Fig. 1(d)]: $\hbar \omega_{1}=1.25 \mathrm{eV}$ and $\hbar \omega_{2}=2.5 \mathrm{eV}$. Taking into account that the duration of both pulses is $\tau_{1}=\tau_{2} \approx 300 \mathrm{fs}$,
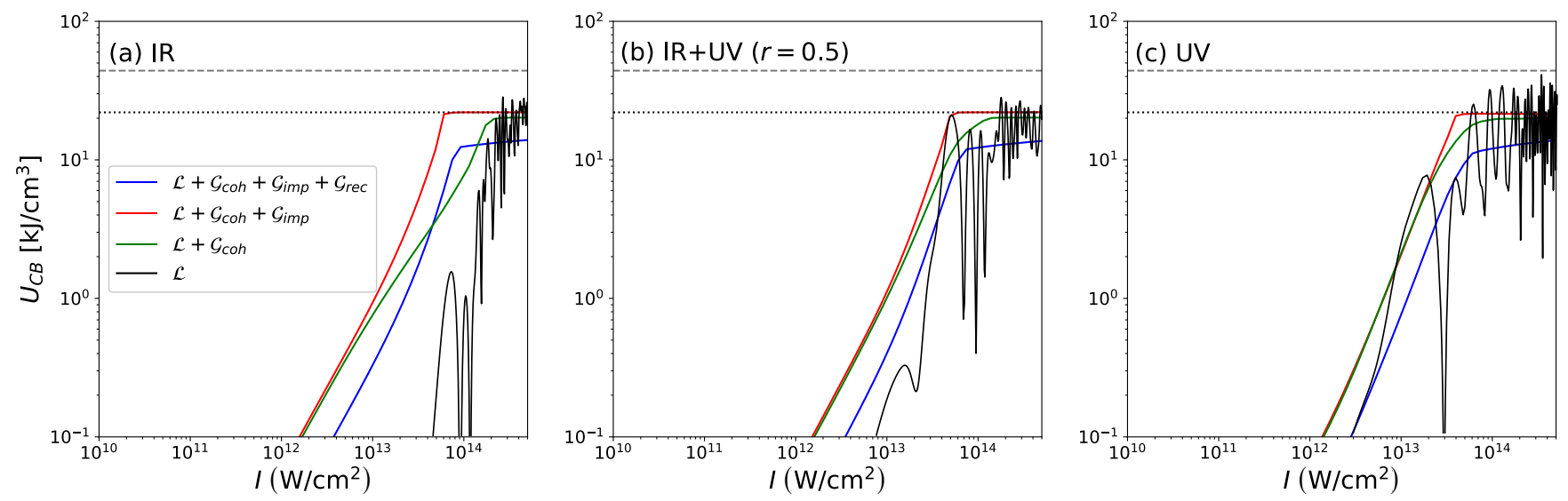

FIG. 8. Energy gained by $\mathrm{CB}$ electrons $U_{\mathrm{CB}}$ as a function of the overall intensity $I$ of the two-color laser of Fig. 1(d), calculated according to different right-hand-side terms in Eq. (1) and several values of $r$ : (a) $r=0$ (pure infrared), (b) $r=0.5$, and (c) $r=1$ (pure ultraviolet). The horizontal dotted line in black represents the energy density of Eq. (9). The maximum energy that the electron subsystem can achieve is depicted by the horizontal dashed line in gray. For the printed grayscale version, the following information allows one to distinguish different curves. (a)-(c) The lines from the bottom to top at $I=10^{12} \mathrm{~W} / \mathrm{cm}^{2}$ correspond to the model version $\mathcal{L}, \mathcal{L}+\mathcal{G}_{\text {coh }}+\mathcal{G}_{\text {imp }}+\mathcal{G}_{\text {rec }}, \mathcal{L}+\mathcal{G}_{\text {coh }}$, and $\mathcal{L}+\mathcal{G}_{\text {coh }}+\mathcal{G}_{\text {imp }}$, respectively. 

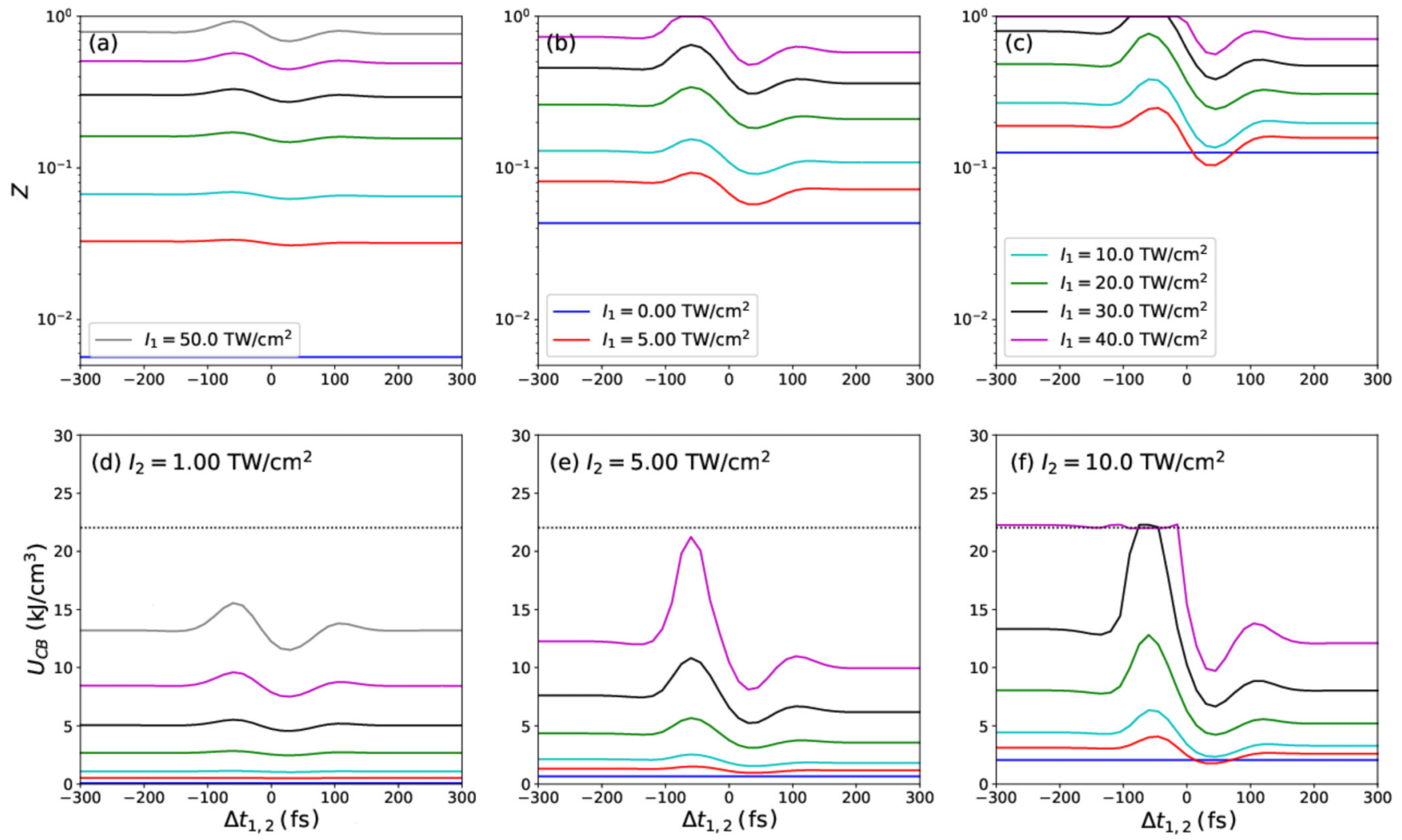

FIG. 9. Ionization degree $Z$ (top row) and energy gained by $\mathrm{CB}$ electrons $U_{\mathrm{CB}}$ (bottom row), calculated at the end of the laser pulse, as a function of the time delay between the IR and UV colors $\Delta t_{1,2}$. Several IR intensities $I_{1}$ (see legend) are added to the UV intensity $I_{2}$ : (a), (d) $I_{2}=10^{12} \mathrm{~W} / \mathrm{cm}^{2}$, (b), (e) $I_{2}=5 \times 10^{12} \mathrm{~W} / \mathrm{cm}^{2}$, and (c), (f) $I_{2}=10^{13} \mathrm{~W} / \mathrm{cm}^{2}$. In (d)-(f) the horizontal dotted line in black represents the energy density of Eq. (9), at which the system saturates. For the printed grayscale version, the following information allows one to distinguish different curves. (a)-(f) The lines from the bottom to top at $\Delta t_{1,2}=-300 \mathrm{fs}$ correspond to $I_{1}=0, I_{1}=5 \mathrm{TW} / \mathrm{cm}^{2}, I_{1}=10 \mathrm{TW} / \mathrm{cm}^{2}, I_{1}=$ $20 \mathrm{TW} / \mathrm{cm}^{2}, I_{1}=30 \mathrm{TW} / \mathrm{cm}^{2}, I_{1}=40 \mathrm{TW} / \mathrm{cm}^{2}$, and $I_{1}=50 \mathrm{TW} / \mathrm{cm}^{2}$, respectively.

the time delay is varied from -300 fs (the UV pulse first excites electrons, and then the IR pulse) to +300 fs. The same operators as in Sec. V are used, with the same parameters, except for the recombination process which has been removed to facilitate the interpretation of the forthcoming results. Similar conclusions are obtained by introducing the electron recombination (results not shown).

Figure 9 shows the ionization degree and the energy gained by conduction electrons as a function of $\Delta t_{1,2}$ and various combinations of values for $I_{1}$ (intensity of IR pulse) and $I_{2}$ (intensity of UV pulse). These values are taken at the upper limit of the time window, i.e., $t=300 \mathrm{fs}$, where these quantities reach their maximum value. Regardless of the intensity couples, the evolution of $Z$ with $\Delta t_{1,2}$ exhibits the same general shape which is (i) constant $Z\left(\Delta t_{1,2}=-300 \mathrm{fs}\right)$, (ii) then $Z$ increases to a maximum value for $\Delta t_{1,2} \simeq-50 \mathrm{fs}$, (iii) decreases to a minimum value for $\Delta t_{1,2} \simeq 50 \mathrm{fs}$, and (iv) reaches another constant $Z\left(\Delta t_{1,2}=300 \mathrm{fs}\right) . Z\left(\Delta t_{1,2}=\right.$ $-300 \mathrm{fs})>Z\left(\Delta t_{1,2}=300 \mathrm{fs}\right)$ which can be explained as follows. For $\Delta t_{1,2}=-300 \mathrm{fs}$, the UV pulse produces (and heats) free electrons which are efficiently heated by the following IR pulse, leading to a given contribution of the impact ionization to the final value of $Z$. For $\Delta t_{1,2}=300 \mathrm{fs}$, first the IR pulse produces less free electrons. Then when the UV pulse heats these electrons less efficiently, the contribution of the impact ionization is smaller, resulting in a smaller final value of $Z$.
For delays of $\sim-100$ fs when both pulses start to overlap significantly, another process takes place, increasing $Z$. For illustration purposes, Fig. 10 shows the electron population in each conduction state (averaged over 50 fs to avoid oscillatinglike behavior, thus better highlighting the main trends) together with the laser electric field with respect to time, for three cases: (a), (d) $\Delta t_{1,2}=-50 \mathrm{fs}$, (b), (e) $\Delta t_{1,2}=0$, and (c), (f) $\Delta t_{1,2}=50$ fs. As shown by Fig. 10(d), up to $t \simeq 150$ fs, the higher the energy level, the smaller is the electron population. Despite the UV pulse only filling odd levels (because $\hbar \omega_{2}=2 \Delta E$ ), the decoherence relaxation leads to the filling of intermediate even states. For a longer time where the maximum IR has been reached, the population of all odd levels is larger than the one of even levels. It may be explained by the fact that the simultaneous absorption of two IR photons strengthens the coupling between odd levels, which becomes stronger than the decoherence relaxation. This cooperative effect leads to more electrons reaching the highest-energy state, thus leading to more efficient impact ionization, and larger $Z$. This cooperative influence vanishes for longer delays because the IR pulse intensity is not high enough to make two-photon absorption when the UV pulse leads to the most efficient heating when its maximum intensity is reached $[t \simeq$ 200 fs in Figs. 10(c) and 10(f)]. We observe that the heating is even less efficient, leading to a low population of electrons in the highest state, and thus an even smaller contribution of 

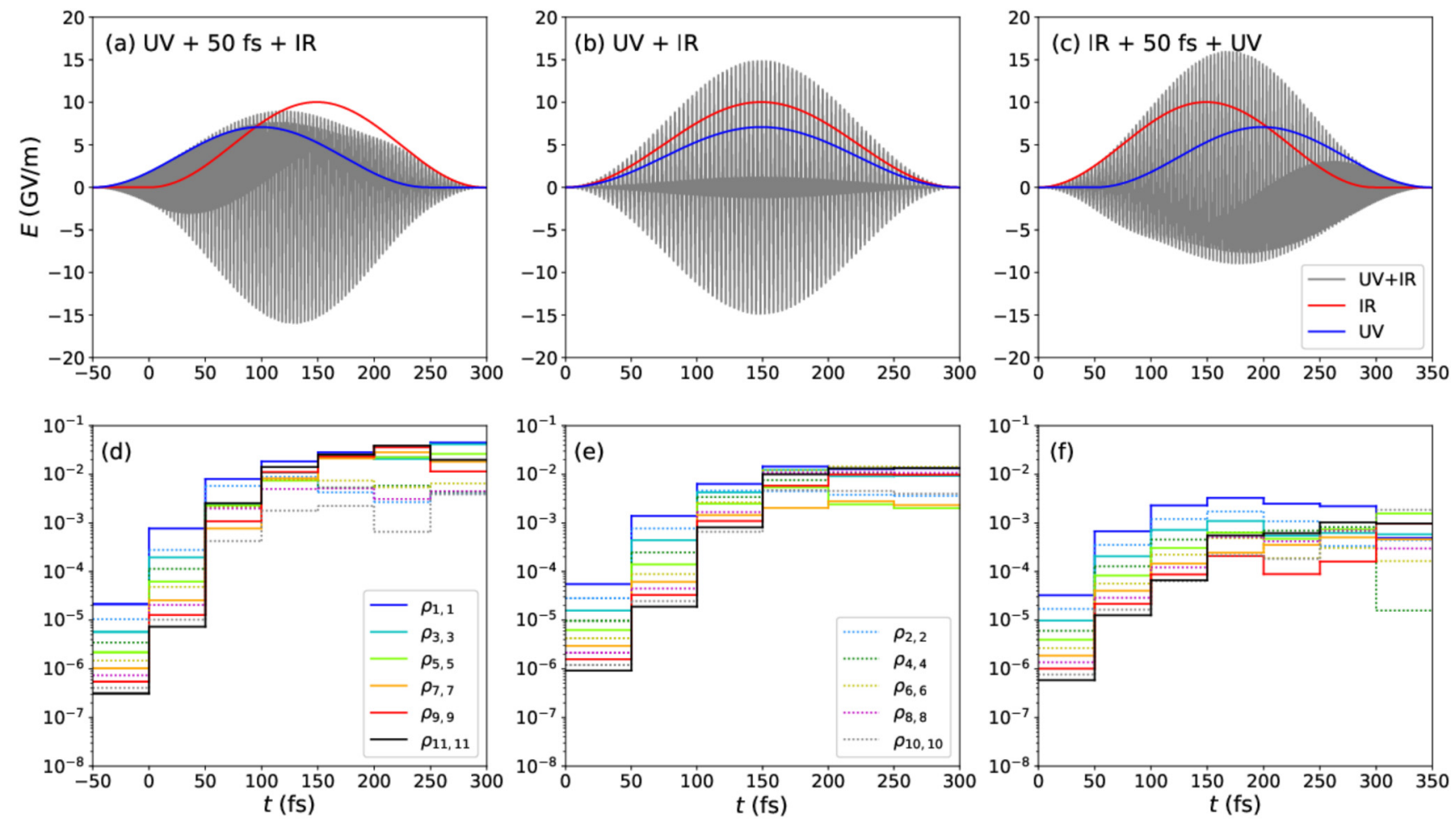

FIG. 10. Top row: Electric field $E(t)$ given by Eq. (5) (gray curves) with the photon energies of Fig. $1(\mathrm{~d})$ and the intensities $I_{2}=10^{13}$ $\mathrm{W} / \mathrm{cm}^{2}$ (UV color) and $I_{1}=2 \times 10^{13} \mathrm{~W} / \mathrm{cm}^{2}$ (infrared). The related square-sine-like slowly varying envelopes $a_{l} \sin ^{2}\left[\pi\left(t-t_{l}\right) / \tau_{l}\right]$ are plotted for each color (see legend). Bottom row: Corresponding electron population in CB levels $\rho_{j, j}$, averaged in time intervals of $50 \mathrm{fs}$ and calculated without recombination. (a), (d) UV comes $50 \mathrm{fs}$ before IR (we consider $t_{2}=-50 \mathrm{fs}$ and $t_{1}=0 \mathrm{fs}$ ). (b), (e) There is no time delay between the two colors (we consider $t_{2}=t_{1}=0 \mathrm{fs}$ ). (c), (f) IR comes $50 \mathrm{fs}$ before UV (we consider $t_{2}=0 \mathrm{fs}$ and $t_{1}=50 \mathrm{fs}$ ). For the printed grayscale version, the following information allows one to distinguish different curves. (d)-(f) The lines from the top to bottom at $t=0$ correspond to $\rho_{1,1}, \rho_{2,2}, \ldots, \rho_{11,11}$, respectively.

the impact ionization, and smaller values of $Z$ for a positive delay. Finally, it can be observed that $Z$ always exhibits a minimum value for $\Delta t_{1,2} \simeq+50$ fs. We do not have a clear explanation for this observation up to now. We emphasize the main result of this study is the enhancement of conduction electron production for $\Delta t_{1,2} \simeq-50 \mathrm{fs}$.

Note that wavelengths of pulses are significantly different so that no interference is expected to take place which may have affected the electron dynamics. However, the temporal total electric field shape can change with respect to the pulseto-pulse delay, i.e., electric field modulations take place (see Fig. 10). Since we observe a rather regular evolution of $Z$ as a function of the delay (Fig. 9), it means such electric field modulations do not play a significant role on the electron dynamics. This is due to the relatively long pulses of $300 \mathrm{fs}$ containing tens of optical cycles (a significant influence could be expected with few cycles pulses). It thus turns out that resonant ionization pathways play the main role for free-electron production, as observed in Refs. [30,36].

Regarding the electron energy density, it mainly mimics the behavior of $Z$ because it linearly depends on each level population. In particular, there is an optimal time delay $\sim-50$ fs to optimize the energy deposition into the material. Note that further simulations have been performed still with reasonable values of characteristic times of 0.5 and 3 fs for the impact ionization (results not shown), with laser intensities of $I_{1}=5 . \times 10^{12} \mathrm{~W} / \mathrm{cm}^{2}$ and $I_{2}=10^{13} \mathrm{~W} / \mathrm{cm}^{2}$ (these intensities lead to an energy deposition close to the material modification threshold which is of interest for application purposes). Obviously the shorter the impact collision time, the larger is the free-electron density, and the larger is the absorbed energy density. The pulse-to-pulse delay optimizing the energy deposition remains of the same order of magnitude as previously (there is a shift of only a few fs). Within the present conditions, this observation can be explained by the fact that the photoionization is the largest contribution to the production of conduction electrons. Other laser intensities in the same range provide the same trends. The influence of the coherence relaxation time from 5 to $20 \mathrm{fs}$, with the same laser intensities close to the energy density threshold, has also been studied (results not shown). Contrary to Sec. IV where the density of conduction electrons is kept constant (no ionization), the shorter the coherence relaxation time, the larger is the absorbed energy density. We have checked this opposite behavior is due to the photoionization and thus the density of excited electrons to the conduction band. Indeed, electrons go back and forth between the valence band and conduction band through photoionization almost reversibly. By adding decoherence, i.e., irreversibility, this back and forth evolution is reduced, leading to a larger production of conduction electrons. The shorter the coherence relaxation time, the larger is the produced conduction electron density, and the larger is the absorbed energy density. However, we do not observe a significant evolution of the pulse-to-pulse optimal 
delay within the standard femtosecond range of coherence relaxation time, indicating that the electron dynamics is driven at first order by the laser-induced direct transition between states.

The prediction of such an optimal delay is in good agreement with experimental observations reported in Ref. [25], where the UV damage threshold is measured as a function of the pulse-to-pulse delay for various given IR fluences. The evolution of the UV damage threshold clearly exhibits a minimum value for a delay $\leqslant 100 \mathrm{fs}$, demonstrating the cooperation of both colors to induce damage, i.e., to induce a certain amount of energy density into the material. Despite different laser parameters being used in the present theoretical study, the orders of magnitude of both the photon energy and pulse duration are such that similar processes are expected to take place. The fact that our OBE model predicts a correct order of magnitude despite different laser parameters (but with the same order of magnitude) is most probably due to the fact that the main effect responsible for the occurrence of this delay is correctly included in our model: the interplay between photoionization and impact ionization. The UV pulse mainly produces the seeded free electrons which are then better heated by the IR pulse leading to impact ionization. For too short delays, not enough seeded electrons are produced. For too long delays, the interplay between both pulses cannot take place, explaining the occurrence of a delay.

\section{CONCLUSION}

Within the framework of the enhancement of the laser energy deposition into dielectric materials by two-color femtosecond laser pulses, a previously developed OBE model has been used to provide an in-depth theoretical understanding of the mechanisms at play during the interaction of such laser pulses with fused silica. Photoionization, electron heating in the conduction band, impact ionization, collisions in the conduction band, and electron recombination are described. In the case where only photoionization takes place, the study of various color configurations has shown how the two pulses can cooperate or not to ionize the material. In particular, due to its nonlinear behavior, it has been evidenced that quantum ionization pathways mixing both photon energies play an important role in setting the density of conduction electrons. On the contrary, since the dynamics of conduction electrons is mainly driven by single-photon absorption, i.e., linear absorption, no specific effect of using two colors is observed, the standard Drude model is able to account for such a configuration. When all processes are allowed with not-delayed pulses, through a detailed examination of the influence of each mechanism, we demonstrate that the approach based on optical Bloch equations succeeds in handling collective effects, both constructive and destructive, due to this two-color configuration. Finally, this model accounts for an optimal delay between pulses which boosts dramatically the energy gained by electrons in the conduction band, owing to electron avalanche seeded from photoionization by an ultraviolet pulse, and heating by an infrared pulse. This observation is in good agreement with experimental observations, thus further validating this theoretical approach. Note that despite particular photon energies having been used in the present paper for modeling purposes, all predicted trends are expected to be general within such laser parameters.

Based on these results, to get a more accurate description of the present physical system to go toward a very quantitative comparison with experimental data, the next step is to improve the present OBE modeling by introducing further physical effects including the contribution of a self-trapped exciton (additional state in the band gap), the dispersion relation of electron bands, a better description of collision frequency with in particular a dependence on the electron energy, the coupling with a Maxwell solver to describe the laser propagation, etc. Through such improvements, the present model may pave the way to a powerful numerical tool to optimize and predict the best configurations for the material modifications driven by temporally shaped laser pulses.

\section{ACKNOWLEDGMENTS}

This research was supported by the project ASTGV (Amplitude Systèmes Through-Glass Via) from French DGA (Direction Générale de l'Armement) funding. Numerical simulations were performed using computing resources at Grand Equipement National pour le Calcul Intensif (GENCI, Grants No. A0030506129 and No. A0040507594). This work was supported by Russian Science Foundation (RSF) (Project No. 17-72-20130). E.S. would like to thank Prof. F. V. Potemkin for helpful communications.

\section{APPENDIX A: PHYSICAL RESTRICTIONS FOR DENSITY MATRIX}

The following notations for algebraic operations on a matrix $A$ are used: (1) conjugation: $A^{*} ;(2)$ inversion: $A^{-1} ;(3)$ transposition: $A^{T}$; (4) conjugate transposition: $A^{\dagger}$.

The density matrix must verify the following physical requirements [35,45]:

(a) Hermiticity:

$$
\hat{\rho}^{\dagger}=\hat{\rho}
$$

(b) unitariness:

$$
\operatorname{Tr}\{\hat{\rho}\}=1 ;
$$

(c) non-negative on-diagonal elements:

$$
0 \leqslant \rho_{j, j} \leqslant 1 ;
$$

(d) Frobenius norm bounded from above by unity:

$$
\|\hat{\rho}\|^{2}=\operatorname{Tr}\left\{\hat{\rho} \hat{\rho}^{\dagger}\right\} \leqslant 1 ;
$$

(e) semidefinite positiveness (non-negative eigenvalues):

$$
\hat{\rho} \geqslant 0 \text {. }
$$

After some manipulations, the Frobenius norm of the density matrix can be expressed as the sum of the contributions of the on-diagonal elements $\left(1-2\|\hat{\rho}\|_{\text {on }}^{2}\right)$ and the off-diagonal elements $\left(2\|\hat{\rho}\|_{\text {off }}^{2}\right)$,

$$
\|\hat{\rho}\|^{2}=1-2\|\hat{\rho}\|_{\text {on }}^{2}+2\|\hat{\rho}\|_{\text {off }}^{2},
$$




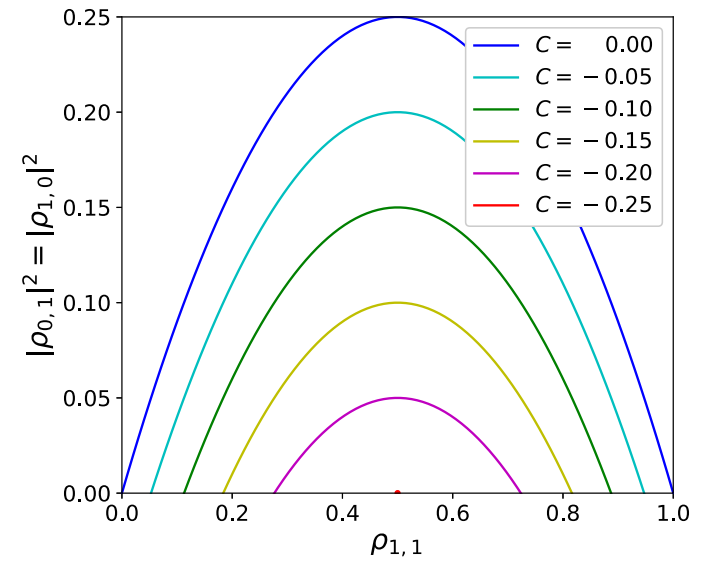

FIG. 11. Equation (A10) applied to the two-level Bloch system: Allowed values of $\left|\rho_{0,1}\right|^{2}$ and $\rho_{1,1}$ according to the value of $C$.

where $\|\hat{\rho}\|_{\text {on }}^{2}$ encompasses the probabilities of populating CB levels,

$$
\|\hat{\rho}\|_{\mathrm{on}}^{2}=Z-\frac{1}{2} Z^{2}-\frac{1}{2} \sum_{j>0} \rho_{j, j}^{2},
$$

and $\|\hat{\rho}\|_{\text {off }}^{2}$ gathers the probabilities of transiting between levels,

$$
\|\hat{\rho}\|_{\text {off }}^{2}=\sum_{k>j}\left|\rho_{j, k}\right|^{2} .
$$

The maximum value that Eq. (A7) reaches is

$$
\max \|\hat{\rho}\|_{\text {on }}^{2}=\frac{N}{2 N+2},
$$

which corresponds to $\rho_{0,0}=\cdots=\rho_{N, N}=1 /(N+1)$.

From Eq. (1) with $\hat{\mathcal{G}}(\hat{\rho})=0$ [see Appendix D for $\hat{\mathcal{G}}(\hat{\rho}) \neq$ $0]$, one deduces that the Frobenius norm is preserved over time: $\partial_{t} \operatorname{Tr}\left\{\hat{\rho} \hat{\rho}^{\dagger}\right\}=0$. Therefore, the time evolution of the density matrix must satisfy

$$
\|\hat{\rho}\|_{\text {off }}^{2}=C+\|\hat{\rho}\|_{\text {on }}^{2},
$$

where $C$ is a constant depending on the suitably chosen initial conditions. Satisfying Eq. (A4) restricts considerably the values of $C$ that make sense from a physical point of view. Taking into account Eq. (A9), we conclude that the constant must lie in the interval

$$
-\frac{N}{2 N+2} \leqslant C \leqslant 0 .
$$

Moreover, according to Eq. (A6), the minimum value that the norm of the density matrix can possess corresponds to $C=-N /(2 N+2)$, which simultaneously minimizes $\|\hat{\rho}\|_{\text {off }}^{2}$ to 0 (i.e., electrons can no longer transit between levels) and maximizes $\|\hat{\rho}\|_{\text {on }}^{2}$ to Eq. (A9). The norm of the density matrix is then not only bounded from above, as stated by Eq. (A4), but also from below:

$$
\frac{1}{N+1} \leqslant\|\hat{\rho}\|^{2} \leqslant 1
$$

As an example, Fig. 11 shows the values that $\left|\rho_{0,1}\right|^{2}=$ $\left|\rho_{1,0}\right|^{2}$ and $\rho_{1,1}$ can have according to the value of the constant
$C$, for the two-level Bloch system $(N+1=2)$. Figure 11 corresponds to the graphical interpretation of Eq. (A10) for two levels. During the evolution of the Bloch system, at every instant the norm of the off-diagonal elements of the density matrix can be deduced from the on-diagonal elements. The constant $C$ appears in that expression, which depends on the initial conditions. In the particular case of two levels, the offdiagonal and on-diagonal norms vary following a parabola. Moving along this curve is equivalent to oscillations of these norms (Rabi oscillations). Initial conditions determine how much the norms of the elements of the density matrix can vary over time: The lower the constant $C$ is, the smaller is the number of degrees of freedom of the Bloch system.

In order to set a physically suitable initial condition for the density matrix, we can proceed as follows. We assign the electron populations to each energy level $\left(\rho_{j, j}\right)$ and choose a value for the constant $C$ according to Eq. (A11). Throughout this paper we use the value $C=0$. Finally, the off-diagonal elements are calculated so that $\|\hat{\rho}\|_{\text {off }}^{2}$ is given from Eq. (A10) and the density matrix is semidefinite positive (diagonallydominant-matrix criterion),

$$
\sum_{k, k \neq j}\left|\rho_{j, k}\right| \leqslant \rho_{j, j},
$$

for each energy level.

\section{APPENDIX B: RESONANT BEHAVIOR OF IONIZATION IN THE TWO-LEVEL BLOCH MODEL}

In this Appendix we aim at justifying why, under the multiphoton ionization (MPI) limit, the output of the two-level Bloch system enters in resonance with the input laser field only when the gap energy is equal to a certain combination of irradiating photons. To do so, we consider that the density matrix reads

$$
\hat{\rho}=\left(\begin{array}{lc}
1-Z_{0}-\tilde{\rho} & \rho_{0,1} \\
\rho_{0,1}^{*} & Z_{0}+\tilde{\rho}
\end{array}\right),
$$

where $0 \leqslant Z_{0} \leqslant 1$ is the initial ionization degree and $\tilde{\rho}=$ $\rho_{1,1}-Z_{0}$, assumed small in the MPI limit, is the laser-induced perturbation of the $\mathrm{CB}$ electron population. According to Eq. (A10), the initial complex module of the off-diagonal element is $\left|\rho_{0,1}(0)\right|=\sqrt{C+Z_{0}-Z_{0}^{2}}$. Without loss of generality, we consider that the following laser field (for $t \geqslant 0$ ),

$$
E(t)=\sum_{j=1}^{\infty} a_{j} \sin \left(j \omega_{0} t\right),
$$

where $\omega_{0}$ is the fundamental pulsation and $a_{j}$ are the electricfield amplitudes. Since the laser is decomposed in commensurable colors, the resonant condition is that $E_{g} /\left(\hbar \omega_{0}\right)=K$ be an integer. After some manipulations, Eq. (1) applied to Eq. (B1) yields

$$
\begin{gathered}
\frac{\partial_{t} \tilde{\rho}}{\omega_{0}}=-2 \frac{e \mu_{0,1} E(t)}{\hbar \omega_{0}} \operatorname{Im} \rho_{0,1}, \\
\frac{\partial_{t} \rho_{0,1}}{\omega_{0}} \approx i K \rho_{0,1},
\end{gathered}
$$

where on the right-hand side of Eq. (B4) the terms depending on $E(t)$ and/or $\tilde{\rho}$, which are 

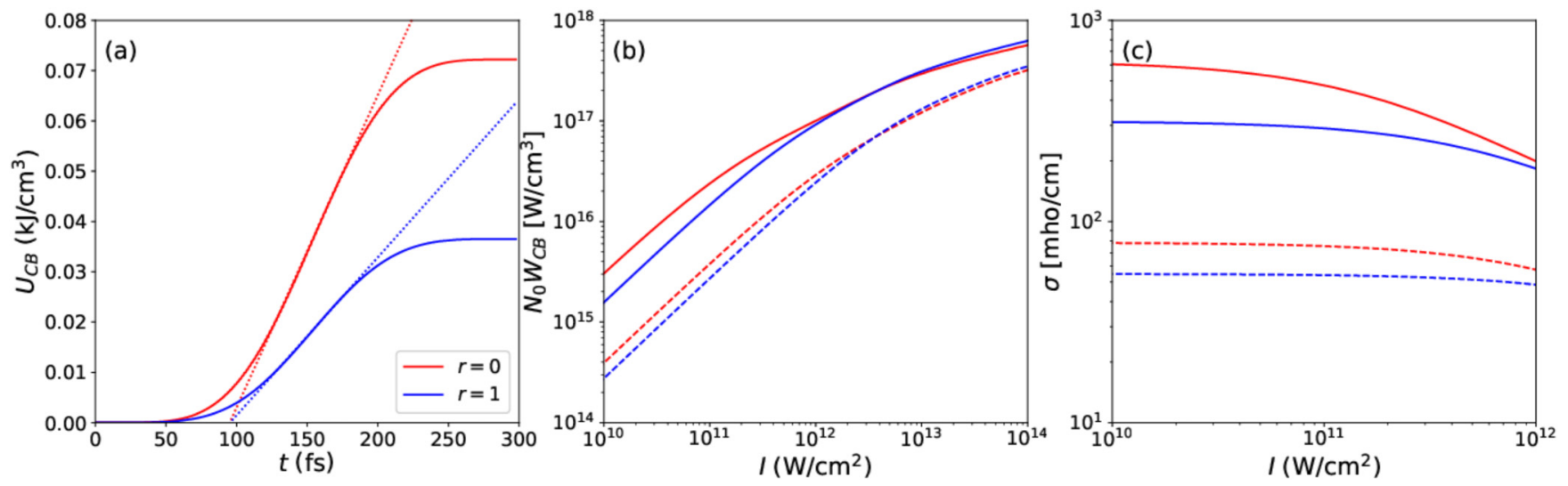

FIG. 12. (a) Example of the energy density gained by CB electrons in the Bloch model (solid curves) as a function of time, calculated following the conditions of Sec. IV with $\tau_{\text {coh }}=10 \mathrm{fs}$. We consider a single-color laser of $a=0.1 \mathrm{GV} / \mathrm{m}\left(I \approx 2 \times 10^{9} \mathrm{~W} / \mathrm{cm}^{2}\right):$ The red curve accounts for pure infrared $(r=0)$, while the blue curve accounts for pure ultraviolet $(r=1)$. The derivative at half maximum (dotted curves), calculated with least squares, is taken as the heating rate (here, $\mathcal{N}_{0} W_{\mathrm{CB}} \approx 0.62 \times 10^{15} \mathrm{~W} / \mathrm{cm}^{3}$ and thus $\sigma \approx 621 \mathrm{mho} / \mathrm{cm}$ for infrared; $\mathcal{N}_{0} W_{\mathrm{CB}} \approx 0.31 \times 10^{15} \mathrm{~W} / \mathrm{cm}^{3}$ and $\sigma \approx 314 \mathrm{mho} / \mathrm{cm}$ for ultraviolet). Simulation starts at $t_{1}=t_{2}=0$ fs. (b) Heating rate as a function of laser intensity for $\tau_{\mathrm{coh}}=10 \mathrm{fs}$ (solid lines) and $\tau_{\mathrm{coh}}=1 \mathrm{fs}$ (dashed lines). Same color legend. (c) Corresponding conductivity of CB electrons. For the printed grayscale version, the following information allows one to distinguish different curves. For every above-mentioned configuration at low intensity, the curve corresponding to $r=0$ is always above the curve corresponding to $r=1$.

$i e\left[\mu_{0,0} \rho_{0,1}+\mu_{0,1}\left(2 Z_{0}-1+2 \tilde{\rho}\right)\right] E(t) /\left(\hbar \omega_{0}\right)$, have been neglected against $i K \rho_{0,1}$ under the MPI limit.

The integration of Eq. (B4) over time gives $\rho_{0,1} \sim$ $\exp \left(i K \omega_{0} t\right)$ and, hence, the complex argument of $\rho_{0,1}$ oscillates at the Rabi pulsation $E_{g} / \hbar=K \omega_{0}$. Furthermore, from Eq. (B3) we have that $\partial_{t} \tilde{\rho} \sim \sin \left(K \omega_{0} t\right) E(t)$. Then it is straightforward to conclude that, provided that $K$ is an integer, the time evolution of the density matrix is periodic with the same fundamental pulsation as the laser field, which means that the Bloch system enters in resonance.

The law $Z \propto I^{K}$ under the MPI regime is obtained by calculating the transition probability for a $K$-photon absorption process $[55,65]$.

\section{APPENDIX C: ENERGY GAINED BY CB ELECTRONS AND DRUDE MODEL}

Let us consider long enough pulses with $\hbar \omega_{1}<\hbar \omega_{2}, \tau_{1}=$ $\tau_{2}$, and $\Delta t_{1,2}=0$, so that we can characterize the laser field in Eq. (5) by the root-mean-square amplitude $a^{2}=a_{1}^{2}+a_{2}^{2}=$ $2 I /\left(n_{0} c \epsilon_{0}\right)$, as in Sec. IV. The Drude model claims that the heating rate $W_{\mathrm{CB}}$ is proportional to the laser intensity $[46,60,61]$,

$$
W_{\mathrm{CB}}=\frac{\sigma}{\mathcal{N}_{0}} a^{2}
$$

where $\sigma$ is the conductivity of $\mathrm{CB}$ electrons. In the literature this conductivity is estimated as follows,

$$
\frac{\sigma}{\mathcal{N}_{0}}=\frac{e^{2} \tau_{\text {coll }}}{3 m_{\mathrm{e}}\left(1+\omega^{2} \tau_{\text {coll }}^{2}\right)},
$$

where $\omega$ is the pulsation of the incident photon, $m_{\mathrm{e}}$ is the mass of free electrons, and $\tau_{\text {coll }}$ is the average collision time of $\mathrm{CB}$ electrons and it is measured to be of the order of $1-10$ fs for fused silica [53,54,61]. Equation (C2) yields $\sigma \approx 57-449 \mathrm{mho} / \mathrm{cm}$ for the infrared photon $\hbar \omega_{1}=1.25 \mathrm{eV}$, and a smaller $\sigma \approx 14-134 \mathrm{mho} / \mathrm{cm}$ for the ultraviolet photon $\hbar \omega_{2}=2.5 \mathrm{eV}$.

In the Bloch model, as shown in Fig. 12(a), the energy density gained by $\mathrm{CB}$ electrons follows an erf-like evolution over time and, hence, the heating rate $\mathcal{N}_{0} W_{\mathrm{CB}}$ is numerically estimated as the time derivative, calculated by least squares, at half maximum, i.e., at the instant when the energy density attains $\left[U_{\mathrm{CB}}(0)+U_{\mathrm{CB}}\left(\tau_{1}\right)\right] / 2$. The heating rates from the Bloch model are plotted in Fig. 12(b), where one sees the behavior $\mathcal{N}_{0} W_{\mathrm{CB}} \propto I$ at low intensities. Following Eq. (C1), the conductivity of $\mathrm{CB}$ electrons yielded by the Bloch model is estimated as

$$
\sigma \approx \frac{\mathcal{N}_{0} W_{\mathrm{CB}}}{a^{2}},
$$

which is plotted in Fig. 12(c). The values obtained from the Bloch model agree with Eq. (C2) within one decade.

Under the Bloch formalism, the energy gained by $\mathrm{CB}$ electrons, defined by Eq. (8) and with the CB band structure presented in Sec. II, can be understood as the expectation value of the operator $\hat{H}_{0}^{+}$[45],

$$
U_{\mathrm{CB}}=\mathcal{N}_{0} \operatorname{Tr}\left\{\hat{H}_{0}^{+} \hat{\rho}\right\},
$$

where $\hat{H}_{0}^{+}$is the positive part of $\hat{H}_{0}$ and it is defined as the diagonal matrix whose on-diagonal elements are the same as those of $\hat{H}_{0}$ if they belong to CB (i.e., $E_{j} \geqslant 0$ ) or zero otherwise. If the electric field amplitude is sufficiently small (MPI regime), in the $\mathrm{CB}$, one-photon absorption implies $\hat{\rho} \propto I$ (see Appendix B), hence Eq. (C4) effectively predicts a heating rate proportional to the laser intensity in our Bloch model.

Finally, by deriving Eq. (C4) with respect to time and taking into account Eq. (1), after some manipulations we obtain the following equation for the energy density gained by $\mathrm{CB}$ electrons,

$$
\begin{aligned}
\partial_{t} U_{\mathrm{CB}}= & -v_{\mathrm{rec}} U_{\mathrm{CB}}-v_{\mathrm{imp}} \mathcal{N}_{0}\left(E_{N}-E_{1}\right) \rho_{N, N} \\
& +\Delta \omega \mathcal{N}_{0} \operatorname{Tr}\{e E \hat{M} \hat{\rho}\}
\end{aligned}
$$


where the operator $\hat{M}$ is

$$
\hat{M}=\frac{i}{\hbar \omega}\left[\hat{H}_{0}^{+}, \hat{\mu}\right] .
$$

Both $C_{\mu}$ and $\tau_{\text {coh }}$ are the key parameters that allow us to control the value of the conductivity in the Bloch model. Indeed, we can see this from Eq. (C5) under the conditions of Sec. IV, i.e., in the absence of VB and thus $v_{\text {imp }}=v_{\text {rec }}=$ 0 and $\hat{H}_{0}^{+}=\hat{H}_{0}$. From $E_{j}-E_{1}=(j-1) \hbar \omega, \mu_{j, k}=\mu_{k, j}=$ $C_{\mu} /\left|E_{j}-E_{k}\right|$, and (C6), we find that $\Delta \omega \hat{M} \propto C_{\mu}$. Therefore, $\sigma \propto C_{\mu}$. The coherence-relaxation time $\tau_{\text {coh }}$, instead, has an indirect influence on $\sigma$. From Eq. (C5) we also have that $\sigma \propto \sum_{k>j>0} \operatorname{Im} \rho_{j, k}$. Since in Appendix D we demonstrated that the norm $\|\hat{\rho}\|$ diminishes in the presence of $\hat{\mathcal{G}}_{\text {coh }}$, so does $\sum_{k>j>0} \operatorname{Im} \rho_{j, k}$. In conclusion, the longer $\tau_{\text {coh }}$ is, the greater $\sigma$ is in our Bloch model, in agreement with Eq. (C2).

\section{APPENDIX D: EQUILIBRIUM STATE OF THE OBE SYSTEM AND CONNECTION WITH RELAXATION PROCESSES}

The system Eq. (1) with $\hat{\mathcal{G}}(\hat{\rho})=0$ enters in equilibrium if it does not vary over time independently of the value of the Hamiltonian $\hat{H}$. It is thus defined as the state that makes $\partial_{t} \hat{\rho}=0$. According to Eq. (2), this state must commute with any value of $\hat{H}$, which is only assured if $\hat{\rho}$ is a scalar matrix (i.e., diagonal matrix whose diagonal elements are all the same value). Therefore, the equilibrium occurs when $\rho_{j, j}=1 /(N+1)$ and $\rho_{j, k \neq j}=0$. This corresponds to $C=-N /(2 N+2)$ in Eq. (A11), which is the lowest possible value of this constant. Furthermore, the norm corresponding to this equilibrium state is the lower bound of Eq. (A12), for which $\|\hat{\rho}\|_{\text {off }}^{2}=0$ (transitions between levels are no longer possible).

From Eq. (1), after some manipulations we can deduce that the relaxation superoperator $\hat{\mathcal{G}}(\hat{\rho})$ affects the preservation of the norm,

$$
\partial_{t} \operatorname{Tr}\left\{\hat{\rho} \hat{\rho}^{\dagger}\right\}=\frac{1}{2} \operatorname{Tr}\{\hat{\rho} \hat{\mathcal{G}}(\hat{\rho})\}
$$

where

$$
\begin{gathered}
\frac{1}{2} \operatorname{Tr}\left\{\hat{\rho} \hat{\mathcal{G}}_{\text {rec }}(\hat{\rho})\right\}=v_{\text {rec }}\left(\|\hat{\rho}\|_{\text {on }}^{2}-\frac{Z}{2}\right), \\
\frac{1}{2} \operatorname{Tr}\left\{\hat{\rho} \hat{\mathcal{G}}_{\text {imp }}(\hat{\rho})\right\}=-v_{\text {imp }} \rho_{N, N}\left(\frac{\rho_{0,0}+\rho_{N, N}}{2}-\rho_{1,1}\right), \\
\frac{1}{2} \operatorname{Tr}\left\{\hat{\rho} \hat{\mathcal{G}}_{\text {coh }}(\hat{\rho})\right\}=-v_{\text {coh }}\|\hat{\rho}\|_{\text {off }}^{2} .
\end{gathered}
$$

Since Eq. (D4) is negative, collisions will always reduce the norm of the density matrix [66] according to Eq. (D1) and, hence, the value of $C$ over time according to Eqs. (A6) and (A10). When $C$ diminishes, the number of degrees of freedom of the system also decreases and therefore the system approaches the state $\rho_{0,0}=\cdots=\rho_{N, N}=1 /(N+1)$ with $C=-N /(2 N+2)$, as we can see in Fig. 11. Precisely, at such a state, Eqs. (D2)-(D4) become zero and, therefore, it is also the equilibrium state for the system Eq. (1) with $\hat{\mathcal{G}}(\hat{\rho}) \neq 0$.

Note that Eqs. (D2) and (D3) might be positive. Since, physically, collisions are present when recombination and impact ionization take place, one must choose carefully the characteristic times of all the relaxation processes so that the norm of the density matrix will not increase.
[1] N. Sanner, O. Utéza, B. Bussiere, G. Coustillier, A. Leray, T. Itina, and M. Sentis, Appl. Phys. A 94, 889 (2009).

[2] B. Chimier, O. Utéza, N. Sanner, M. Sentis, T. Itina, P. Lassonde, F. Légaré, F. Vidal, and J. C. Kieffer, Phys. Rev. B 84, 094104 (2011).

[3] C. Dowding and A. Borman, in Laser Surface Engineering, edited by J. Lawrence and D. Waugh, Woodhead Publishing Series in Electronic and Optical Materials (Woodhead Publishing, Cambridge, UK, 2015), pp. 523-546.

[4] M. C. Gower, Opt. Express 7, 56 (2000).

[5] H. Hocheng, H. Tsai, U. Jadhav, K. Wang, and T. Lin, in Comprehensive Materials Processing, edited by S. Hashmi, G. F. Batalha, C. J. V. Tyne, and B. Yilbas (Elsevier, Oxford, UK, 2014), pp. 75-113.

[6] N. Bellini, R. Geremia, S. Norval, G. Fichet, and D. Karnakis, J. Laser Micro/Nanoeng. 11, 388 (2016).

[7] H. Ma, R. A. Zakoldaev, A. Rudenko, M. M. Sergeev, V. P. Veiko, and T. E. Itina, Opt. Express 25, 33261 (2017).

[8] H. Zheng, Y. Guan, X. Wang, and Z. Wang, in Laser Surface Engineering (Ref. [3]), pp. 317-357.

[9] Q. Ding, L. Wang, and L. Hu, in Laser Surface Engineering (Ref. [3]), pp. 405-422.

[10] A. Rudenko, J.-P. Colombier, S. Hohm, A. Rosenfeld, J. Kruger, J. Bonse, and T. E. Itina, Sci. Rep. 7, 12306 (2017).
[11] A. Papadopoulos, E. Skoulas, G. D. Tsibidis, and E. Stratakis, Appl. Phys. A 124, 146 (2018).

[12] Y. Kawamura, K. Toyoda, and S. Namba, Appl. Phys. Lett. 40, 374 (1982).

[13] R. Srinivasan and V. Mayne-Banton, Appl. Phys. Lett. 41, 576 (1982).

[14] M. Watson, Circuit World 11, 13 (1984).

[15] J. J. Chang, B. E. Warner, E. P. Dragon, and M. W. Martinez, J. Laser Appl. 10, 285 (1998).

[16] N. Bulgakova, R. Stoian, A. Rosenfeld, I. Hertel, W. Marine, and E. Campbell, Appl. Phys. A 81, 345 (2005).

[17] N. M. Bulgakova, V. P. Zhukov, Y. P. Meshcheryakov, L. Gemini, J. Brajer, D. Rostohar, and T. Mocek, J. Opt. Soc. Am. B 31, C8 (2014).

[18] N. M. Bulgakova, V. P. Zhukov, S. V. Sonina, and Y. P. Meshcheryakov, J. Appl. Phys. 118, 233108 (2015).

[19] I. Mirza, N. M. Bulgakova, J. Tomastik, V. Michalek, O. Haderka, L. Fekete, and T. Mocek, Sci. Rep. 6, 39133 (2016).

[20] M. V. Shugaev, C. Wu, O. Armbruster, A. Naghilou, N. Brouwer, D. S. Ivanov, T. J.-Y. Derrien, N. M. Bulgakova, W. Kautek, B. Rethfeld et al., MRS Bull. 41, 960 (2016).

[21] R. Stoian, M. Boyle, A. Thoss, A. Rosenfeld, G. Korn, I. V. Hertel, and E. E. B. Campbell, Appl. Phys. Lett. 80, 353 (2002).

[22] J. Hernandez-Rueda, J. Siegel, M. Galvan-Sosa, A. R. de la Cruz, and J. Solis, J. Opt. Soc. Am. B 30, 1352 (2013). 
[23] Y. P. Deng, X. H. Xie, H. Xiong, Y. X. Leng, C. F. Cheng, H. H. Lu, R. X. Li, and Z. Z. Xu, Opt. Express 13, 3096 (2005).

[24] K. Gaudfrin, J. Lopez, K. Mishchik, L. Gemini, R. Kling, and G. Duchateau, Opt. Express 28, 15189 (2020).

[25] X. Yu, Q. Bian, B. Zhao, Z. Chang, P. B. Corkum, and S. Lei, Appl. Phys. Lett. 102, 101111 (2013).

[26] I. Chowdhury, X. Xu, and A. Weiner, Appl. Phys. A 83, 49 (2006).

[27] S. Höhm, M. Herzlieb, A. Rosenfeld, J. Krüger, and J. Bonse, Appl. Phys. Lett. 103, 254101 (2013).

[28] X. Yu, Q. Bian, Z. Chang, P. B. Corkum, and S. Lei, Opt. Express 21, 24185 (2013).

[29] B. Zhou, A. Kar, M. J. Soileau, and X. Yu, Opt. Lett. 45, 1994 (2020).

[30] J. Liao and J. R. Gulley, J. Opt. Soc. Am. B 31, 2973 (2014).

[31] N. M. Bulgakova, V. P. Zhukov, A. R. Collins, D. Rostohar, T. J.-Y. Derrien, and T. Mocek, Appl. Surf. Sci. 336, 364 (2015).

[32] L. Gallais, D.-B. Douti, M. Commandré, G. Batavičiūte, E. Pupka, M. Ščiuka, L. Smalakys, V. Sirutkaitis, and A. Melninkaitis, J. Appl. Phys. 117, 223103 (2015).

[33] L. V. Keldysh, Zh. Eksp. Teor. Fiz. 47, 1945 (1964) [Sov. Phys. JETP 20, 1307 (1965)].

[34] B. Rethfeld, Phys. Rev. Lett. 92, 187401 (2004).

[35] A. Bourgeade and G. Duchateau, Phys. Rev. E 85, 056403 (2012).

[36] J. R. Gulley and T. E. Lanier, Proc. SPIE 9632, 96320X (2015).

[37] A. N. Pfeiffer, J. Phys. B: At., Mol. Opt. Phys. 53, 164002 (2020).

[38] E. Smetanina, P. González de Alaiza Martínez, I. Thiele, B. Chimier, A. Bourgeade, and G. Duchateau, Phys. Rev. E 101, 063206 (2020).

[39] G. Duchateau, B. Chimier, S. Coudert, E. Smetanina, L. Barilleau, N. Fedorov, H. Jouin, G. Geoffroy, P. Martin, and V. T. Tikhonchuk, Phys. Rev. B 102, 024305 (2020).

[40] E. Gamaly, Phys. Rep. 508, 91 (2011).

[41] R. W. Boyd, Nonlinear Optics, 3rd ed. (Academic, San Diego, CA, 2008).

[42] S. Y. Kruchinin, F. Krausz, and V. S. Yakovlev, Rev. Mod. Phys. 90, 021002 (2018).

[43] B. Bidégaray-Fesquet, Hiérarchie De Modèles En Optique Quantique: De Maxwell-Bloch à Schrödinger Non-Linéaire, 1st ed. (Springer, Berlin, 2006).

[44] B. Bidégaray, A. Bourgeade, and D. Reignier, J. Comput. Phys. 170, 603 (2001).
[45] M. Berman and R. Kosloff, Comput. Phys. Commun. 63, 1 (1991).

[46] B. C. Stuart, M. D. Feit, S. Herman, A. M. Rubenchik, B. W. Shore, and M. D. Perry, Phys. Rev. B 53, 1749 (1996).

[47] B. Rethfeld, D. S. Ivanov, M. E. Garcia, and S. I. Anisimov, J. Phys. D: Appl. Phys. 50, 193001 (2017).

[48] S. S. Nekrashevich and V. A. Gritsenko, Phys. Solid State 56, 207 (2014).

[49] A. Q. Wu, I. H. Chowdhury, and X. Xu, Phys. Rev. B 72, 085128 (2005).

[50] P. Audebert, P. Daguzan, A. Dos Santos, J. C. Gauthier, J. P. Geindre, S. Guizard, G. Hamoniaux, K. Krastev, P. Martin, G. Petite et al., Phys. Rev. Lett. 73, 1990 (1994).

[51] S. Tzortzakis, L. Sudrie, M. Franco, B. Prade, A. Mysyrowicz, A. Couairon, and L. Bergé, Phys. Rev. Lett. 87, 213902 (2001).

[52] J. Rolle, L. Bergé, G. Duchateau, and S. Skupin, Phys. Rev. A 90, 023834 (2014).

[53] D. Arnold, E. Cartier, and D. J. DiMaria, Phys. Rev. B 45, 1477 (1992).

[54] Q. Sun, H. Jiang, Y. Liu, Z. Wu, H. Yang, and Q. Gong, Opt. Lett. 30, 320 (2005).

[55] P. Lambropoulos, in Advances in Atomic and Molecular Physics (Academic, San Diego, CA, 1976), Vol. 12, pp. 87-164.

[56] V. V. Temnov, K. Sokolowski-Tinten, P. Zhou, A. ElKhamhawy, and D. von der Linde, Phys. Rev. Lett. 97, 237403 (2006).

[57] S. Sato, M. Lucchini, M. Volkov, F. Schlapfer, L. Gallmann, U. Keller, and A. Rubio, Phys. Rev. B 98, 035202 (2018).

[58] R. Gattass and E. Mazur, Nat. Photonics 2, 219 (2008).

[59] A. Pineau, B. Chimier, S. X. Hu, and G. Duchateau, Phys. Plasmas 27, 092703 (2020).

[60] B. C. Stuart, M. D. Feit, A. M. Rubenchik, B. W. Shore, and M. D. Perry, Phys. Rev. Lett. 74, 2248 (1995).

[61] T. Q. Jia, H. X. Chen, M. Huang, F. L. Zhao, X. X. Li, S. Z. Xu, H. Y. Sun, D. H. Feng, C. B. Li, X. F. Wang et al., Phys. Rev. B 73, 054105 (2006).

[62] H. L. Schick, Chem. Rev. 60, 331 (1960).

[63] G. Duchateau, C. Illescas, B. Pons, E. Cormier, and R. Gayet, J. Phys. B: At., Mol. Opt. Phys. 33, L571 (2000).

[64] S. Y. Kruchinin, Phys. Rev. A 100, 043839 (2019).

[65] H. B. Bebb and A. Gold, Phys. Rev. 143, 1 (1966).

[66] D. Du, X. Liu, and G. Mourou, Appl. Phys. B 63, 617 (1996). 